\title{
Reliable probabilistic determination of membership in stellar kinematic groups in the young disk ${ }^{\star}$
}

\author{
A. Klutsch ${ }^{1,2}$, R. Freire Ferrero ${ }^{3}$, P. Guillout ${ }^{3}$, A. Frasca ${ }^{1}$, E. Marilli ${ }^{1}$, and D. Montes ${ }^{2}$ \\ 1 INAF - Osservatorio Astrofisico di Catania, via S. Sofia 78, 95123 Catania, Italy \\ e-mail: alexis.klutsch@oact.inaf.it \\ 2 Departamento de Astrofísica, Facultad de Ciencias Físicas, Universidad Complutense de Madrid, 28040 Madrid, Spain \\ 3 Observatoire Astronomique, Université de Strasbourg \& CNRS, UMR 7550, 11 rue de l'Université, 67000 Strasbourg, France
}

Received 30 August 2013 / Accepted 24 March 2014

\section{ABSTRACT}

\begin{abstract}
Context. Over the last century, the overdensities in the velocity distributions of nearby stars were attributed to stellar kinematic groups or moving groups. Although their reality was initially questioned, their existence is now supported by a confluence of evidence. Aims. To pursue investigations, quantitative methods should be clearly defined to identify new stars belonging to these groups. Here, we present two probabilistic methods to determine the likelihood of kinematic membership for possible candidates in five of the known young stellar kinematic groups - namely, Pleiades, IC 2391, Castor, Ursa Major, and Hyades - in which all are younger than 650 Myr. Methods. We tried different methods to handle kinematic data of their known members. We succeeded in developing two independent procedures able to identify new candidates of these five stellar stream. We tested the robustness of our two approaches by means of extensive Monte Carlo simulations.

Results. Our methods are consistent with one another in more than $90 \%$ of cases and for almost all the stellar kinematic groups under scrutiny. The IC 2391 supercluster is an exception. Applying our statistical methods to a large sample of young low-mass stars, we confirmed almost all the likely members and good candidates of these stellar streams. We also proposed 39 additional candidates based on the agreement and the high likelihood of age and kinematic membership.

Conclusions. These probabilistic methods are very powerful to reliably identify new candidate members of young stellar kinematic groups. However, the kinematic criteria alone are not sufficient to distinguish between coeval stars that are evaporated from open clusters and other field stars trapped by dynamical processes generated by galactic perturbations. The identification of stars belonging to the remnant of a past star-forming event can be possible with the help of additional information, such as indicators of chromospheric activity, age proxies (lithium abundance), and chemical composition.
\end{abstract}

Key words. stars: kinematics and dynamics - methods: data analysis - stars: low-mass

\section{Introduction}

One of the pillars of astronomy is the study of the dynamics and evolution of the Milky Way's spiral structure. Currently, additional constraints on non-axisymmetric models are addressed by improving our knowledge on the origin of overdensities found in the velocity distribution of nearby stars. These overdensities define groups of stars, which are commonly called stellar kinematic groups (SKGs). But what are they? Over time, our knowledge of these groups has been subject to profound changes.

The beginning of this research field dates back to the midnineteenth century when Mädler (1846) discovered stars sharing the same Galactic motion across the sky in the Pleiades stellar cluster. Later, Proctor (1869) also identified several stars moving together in two other areas, the Ursa Major and Hyades clusters. Afterward, main contributions in this field were given from Olin Eggen who discovered a dozen other similar groups with a wide range in age (e.g., Eggen 1994, 1996, and references therein). The discovery of overdensities in the velocity distribution of nearby stars led him to introduce two new stellar aggregates, namely "supercluster" (SC) and "moving group" (MG), which are both defined as a clump of stars that are gravitationally unbound. The SC members share the same kinematics and may

* Table 4 and Appendices are available in electronic form at http://www. aanda.org occupy extended regions through the Galaxy. Inside the solar neighborhood, such stellar groups are referred to as MGs whose members appear distributed over all the sky due to their proximity to the Sun. Moreover, each MG can be seen as a unique vestige of a past star formation event, and its members should therefore have a common origin. At the end of the twentieth century, their existence (especially that of the oldest ones) was a matter of concern because the Galactic differential rotation tends to spread the stars and their velocity dispersion increases with age under the disk heating effect (e.g., Griffin 1998; Taylor 2000). Thanks to the astrometric data of the HIPPARCOS space observatory and radial velocity measurements from large spectroscopic surveys, Famaey et al. (2005) closed this debate and finally confirmed the existence of main SKGs.

The application of several numerical methods on the HIPPARCOS data confirmed the reality of both classical young and old structures and even identified some new SKGs along with some substructures within existing ones (see e.g., Dehnen 1998; Chereul et al. 1998, 1999; Skuljan et al. 1999; Torra et al. 2000; Feltzing \& Holmberg 2000; Bovy et al. 2009). Therefore, the Pleiades stream turned out to be a mixture of several subgroups with slightly different ages and kinematical properties (Asiain et al. 1999). During the last decade, many nearby young associations have been discovered from observations in the Southern hemisphere (Zuckerman \& Song 2004; Torres et al. 2008). Their kinematics is rather similar to that of Local 
association. Eggen (1973a) and Eggen \& Iben (1988) denoted the locus of the UVW space that encompasses all these SKGs as the very young disk. From the analysis of the RasTyc sample (Guillout et al. 2009; Frasca et al., in prep.), four comoving $\mathrm{T}$ Tauri stars were recently discovered in a sky region of few degrees toward the CO Cepheus void (Guillout et al. 2010). Klutsch et al. (2011b) extended the member list by the identification of additional young stars in the sky area that surrounds this stellar group. Since all of them share the same proper motion, radial velocity, and age, they likely form the first young association found northward of $\delta=+30 \mathrm{deg}$ (Klutsch et al. 2012; Klutsch et al., in prep.).

Although the existence of main SKGs is no longer questionable, their origin continues to be at the forefront of scientific debate due to the heterogeneity of their members (Dehnen 1998; Chereul et al. 1998, 1999; Famaey et al. 2008; López-Santiago et al. 2009). A considerable number of works has attempted to address this concern. Two explanations are possible: either these structures are the remnants of evaporated clusters or they are created by dynamical processes. Bovy \& Hogg (2010) concluded that none of the moving groups can be the result of the evaporation of a single open cluster. Nevertheless, at least some of the sources that share the same space motion must have a common origin because of the similarity of their age and abundances. In particular, the chemical tagging of the Hyades stream identified several stars that could likely belong to the eponymous open cluster (de Silva et al. 2011; Pompéia et al. 2011; Tabernero et al. 2012).

Recent theoretical approaches (e.g., Quillen 2003; Quillen \& Minchev 2005; Chakrabarty 2007; Minchev \& Quillen 2007; Antoja et al. 2009; Minchev et al. 2010; Minchev \& Famaey 2010; McMillan 2013) have been tested to identify the dominant mechanism that drives the evolution of the velocity distribution of the stars in the solar neighborhood. In their models, they investigated the cases of the resonances that would be caused by either the Galactic bar, the spiral structure, or both. Several recent results favor non-axisymmetric perturbations of the Galactic potential (e.g., Minchev \& Famaey 2010, and references therein). Up to now, none of the proposed processes can explain the formation of all the SKGs. Sellwood $(2010,2011)$ explored the hypothesis of recurrent short-lived spiral transients. De Simone et al. (2004) already showed that the observational features could be reproduced from a succession of strong spiral transients.

Within the locus of the young-disk population defined by Eggen (1973a,b, 1989), many SKGs younger than $650 \mathrm{Myr}$ were detected. In this paper, we focused on the five major ones: namely, the Local association (LA) or Pleiades MG (10$300 \mathrm{Myr}$ ); the IC $2391 \mathrm{SC}$ (80-250 Myr); the Castor MG ( $200 \mathrm{Myr}$ ); the Ursa Major (UMa) MG or Sirius SC (300$500 \mathrm{Myr})$; and the Hyades SC ( $650 \mathrm{Myr})$. The age ranges were taken from López-Santiago et al. (2009). We selected these stellar streams because there are several studies confirming their existence and identifying many of their members. For describing each of the aforementioned SKGs, we used the late-type stars, as listed by Montes et al. (2001), which satisfy one of the two main Eggen's criteria on peculiar and predicted radial velocities. To find new stars belonging to these five kinematic groups, we developed two statistical procedures to determine the likelihood of kinematic membership for them.

The paper is organized as follows. We describe our two probabilistic methods in Sect. 2. For this purpose, we display two different views of the geometrical representation of the kinematic space. Considering each MG as a centrally condensed ellipsoid in the kinematic velocity space (Sect. 2.1), we performed the analysis by taking the inhomogeneity and complexity of each stellar stream (Sect. 2.2) into account. We present our results and discuss them in Sect. 3. For testing the reliability of one of our techniques with respect to the other, we constructed two separate samples (members and non-members) using Monte Carlo simulations (Sect. 3.1). We note that the most sophisticated procedure catches possible candidates that are close to the outer SKG boundaries (Sect. 3.2). In Sect. 4, we applied our procedures on the sample of young low-mass stars listed by Shkolnik et al. (2012) by confirming almost all their likely members and good candidates of these stellar streams. The conclusions are outlined in Sect. 5.

\section{Probabilistic methods for the determination of the likelihood of kinematic membership in an SKG}

The search for new members of close young associations and SKGs was done earlier by using mainly qualitative methods, such as the Eggen's criteria (Eggen 1958, 1995). To gain in objectivity, we determine the membership by means of probabilistic procedures using the kinematic properties of known SKG members (Klutsch 2008; Klutsch et al. 2011a). We tried different methods to handle kinematic data, and we succeeded to develop two independent procedures (Sects. 2.1 and 2.2) to be able to identify new candidates of five young MGs. We widely used $n$-dimensional normal Gaussian distributions to determine the kinematic membership probabilities of each stellar candidate (Appendix A).

Recent works also evaluate the likelihood of membership based on a probabilistic way. Shkolnik et al. (2012) evaluate a kinematic membership probability in 14 young MGs (including those investigated here) by assuming that their reduced $\bar{\chi}^{2}$ follows a typical $\chi^{2}$ statistic distribution with three degrees of freedom. For every highly probable kinematic candidate, the age and position on the sky are then used to improve the final classification. In other recent works, Malo et al. (2013) and Gagné et al. (2014), who were searching for new low-mass members of nearby young associations (Zuckerman \& Song 2004; Torres et al. 2008), computed a probability by means of a Bayesian analysis of stellar properties (i.e., position, proper motion, magnitude, and color) and by adding more observables, such as the radial velocity and distance, in some specific cases.

\subsection{First method: viewing an MG as a continuous ellipsoidal shape (CES) in the kinematic velocity space}

In the space defined by the three velocity components $U, V$, and $W^{1}$, the SKG members are scattered as a cluster of points. The aim of this method is to firstly perform an evaluation in the simplest way, for example, by disregarding the cross terms that are taken into account in our second method (see Sect. 2.2). The only requirement is that the projections of velocities along the $(U, V, W)$ axes are symmetrically distributed. This can also be achieved with an ellipsoidal distribution tilted with the respect of the coordinate system. For further analysis of each SKG, we fitted the distribution of each velocity component with a Gaussian (Fig. 1) to determine their velocity means $\left(U_{0}, V_{0}, W_{0}\right)$

\footnotetext{
The heliocentric space velocity components $(U, V, W)$ have been calculated in the left-handed coordinate system where $U$ is pointing toward the Galactic anticenter, $V$ in the Galactic rotation direction, and $W$ toward the north Galactic pole, respectively.
} 
A. Klutsch et al.: Reliable probabilistic determination of membership in stellar kinematic groups in the young disk
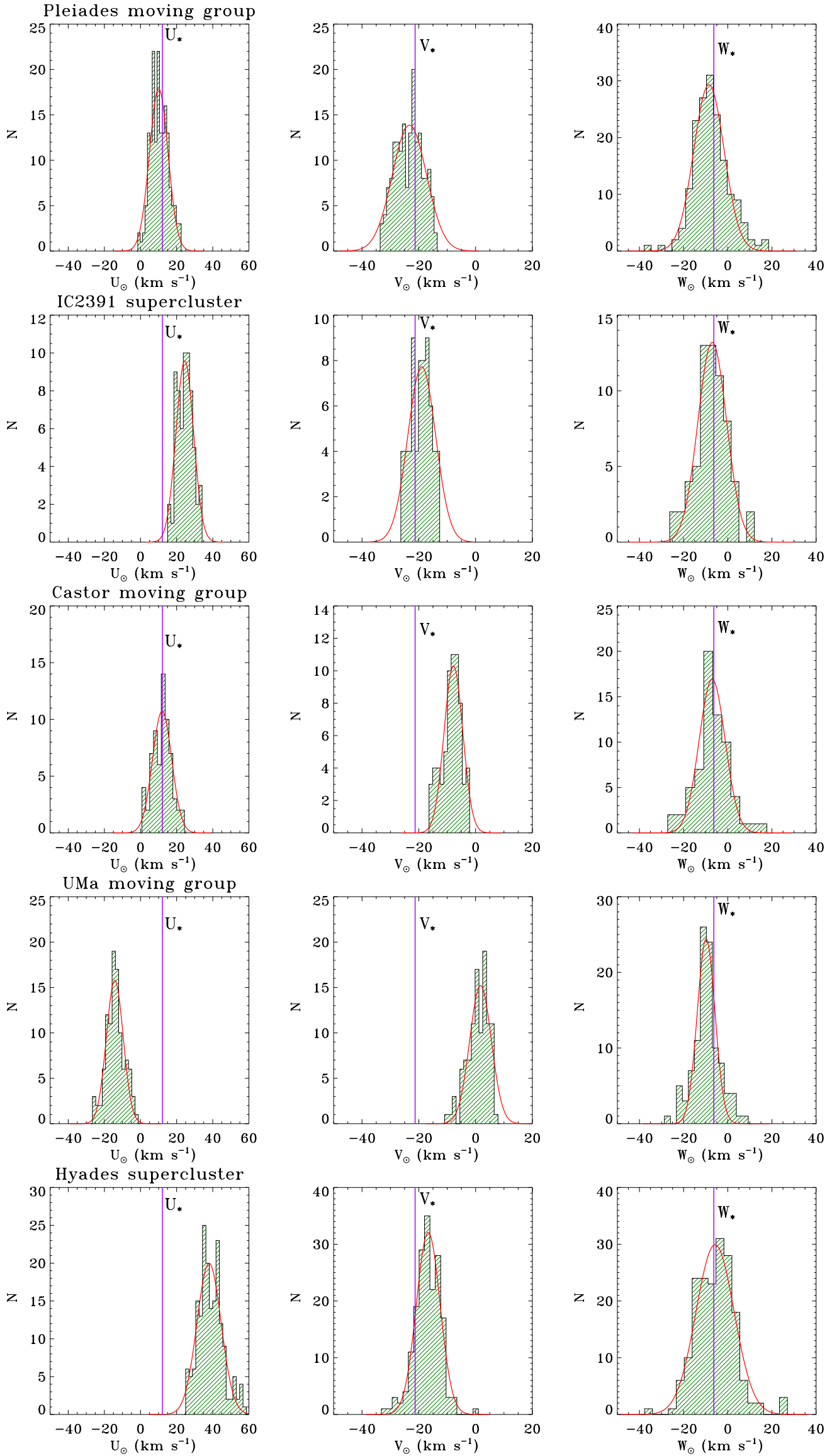

Fig. 1. $U, V$, and $W$ velocities (left to right panels) taken from Montes et al. (2001) for known members of all SKGs studied here. The red curves show the best Gaussian fit of each velocity distribution, as listed in Table 1. The purple vertical lines denote a highly probable kinematic candidate of the Pleiades SKG $\left(P_{3 \mathrm{D}} \sim 95 \%\right.$ and $\left.P_{2 \mathrm{D}} \sim 98 \%\right)$ with velocity components $\left(U_{\star}, V_{\star}, W_{\star}\right)=(12.1,-21.3,-6.3) \mathrm{km} \mathrm{s}^{-1}$. 
Table 1. Gaussian fits (mean and dispersion parameters) of spacevelocity distributions of components $U, V$, and $W$ of known SKG members, defined in the left-handed coordinate system.

\begin{tabular}{lrrrrrr}
\hline \hline SKGs & \multicolumn{1}{c}{$\begin{array}{l}U_{0} \\
\left(\mathrm{~km} \mathrm{~s}^{-1}\right)\end{array}$} & \multicolumn{1}{c}{$\begin{array}{c}\sigma_{0} \\
\left(\mathrm{~km} \mathrm{~s}^{-1}\right)\end{array}$} & \multicolumn{2}{c}{$\begin{array}{l}\sigma_{0} \\
\left(\mathrm{~km} \mathrm{~s}^{-1}\right)\end{array}$} \\
\hline Pleiades MG & 9.8 & 5.1 & -22.9 & 6.0 & -9.2 & 6.2 \\
IC 2391 SC & 23.9 & 3.8 & -18.9 & 4.9 & -8.0 & 5.6 \\
Castor MG & 10.7 & 6.5 & -7.5 & 4.4 & -8.8 & 4.2 \\
UMa MG & -14.4 & 3.1 & 2.1 & 3.3 & -9.6 & 3.6 \\
Hyades SC & 38.0 & 6.6 & -16.9 & 4.2 & -4.7 & 7.1 \\
\hline
\end{tabular}

and dispersion parameters $\left(\sigma_{U}, \sigma_{V}, \sigma_{W}\right)$. All these variables are listed in Table 1 . We noticed that the ellipsoids of velocities for known members used here are mostly aligned with the $(U, V$, $W$ ) axes (see Figs. B.6 to B.10).

We then assume, as a first approximation, that the members of an SKG are distributed in the velocity space within a centrally condensed ellipsoid that we can describe by a threedimensional (3D) normal distribution. This allows us to analytically treat the velocity distribution as a continuous function and to use smoothed density gradients. We then defined the various ellipsoidal contour surfaces on their 3D distribution by mapping the parameter,

$k=\sqrt{\left(\frac{U-U_{0}}{\sigma_{U}}\right)^{2}+\left(\frac{V-V_{0}}{\sigma_{V}}\right)^{2}+\left(\frac{W-W_{0}}{\sigma_{W}}\right)^{2}}$.

According to the locus of a candidate $\left(U_{\star}, V_{\star}, W_{\star}\right)$ on these isosurfaces, we can compute its kinematic membership probability using the following relation (see Appendix A):

$P_{3 \mathrm{D}}=-2 \Phi\left(k_{\star}\right)+\sqrt{\frac{2}{\pi}} k_{\star} \mathrm{e}^{-0.5 k_{\star}^{2}}$

where $\Phi$ is the cumulative distribution function of the standard one-dimensional normal distribution $\mathcal{N}(0,1)$ and $k_{\star}$ corresponds to the normalized velocity modulus of the vector between the center of the SKG distribution and the representative point of the candidate. The parameter $k_{\star}$ is equal to $\sqrt{\left(\frac{U_{\star}-U_{0}}{\sigma_{U}}\right)^{2}+\left(\frac{V_{\star}-V_{0}}{\sigma_{V}}\right)^{2}+\left(\frac{W_{\star}-W_{0}}{\sigma_{W}}\right)^{2}}$. As the membership probability $P_{3 \mathrm{D}}$ depends only on $k_{\star}$, we can estimate its error (Fig. A.2) as follows:

$\Delta P_{3 \mathrm{D}}=\left|\frac{\partial P_{3 \mathrm{D}}}{\partial k_{\star}}\right| \Delta k_{\star}=\sqrt{\frac{2}{\pi}} k_{\star}^{2} \mathrm{e}^{-0.5 k_{\star}^{2}} \Delta k_{\star}$.

The uncertainty of $k_{\star}$, which depends on the error associated to the kinematic of the candidate $\left(\Delta U_{\star}, \Delta V_{\star}, \Delta W_{\star}\right)$, has been obtained by error propagation of Eq. (1):

$$
\begin{aligned}
\Delta k_{\star}^{2}= & \left(\frac{U_{\star}-U_{0}}{\sigma_{U}^{2} k_{\star}}\right)^{2} \Delta U_{\star}^{2}+\left(\frac{V_{\star}-V_{0}}{\sigma_{V}^{2} k_{\star}}\right)^{2} \Delta V_{\star}^{2} \\
& +\left(\frac{W_{\star}-W_{0}}{\sigma_{W}^{2} k_{\star}}\right)^{2} \Delta W_{\star}^{2} .
\end{aligned}
$$

We considered a likelihood of kinematic membership greater than or equal to the threshold of $10 \%$ as appropriate to justify that a star is a new member of a stellar stream. This corresponds to a $k$ threshold of 2.5 for a 3D normal distribution, as indicated in Fig. A.2. For the sake of readability, we illustrate the result

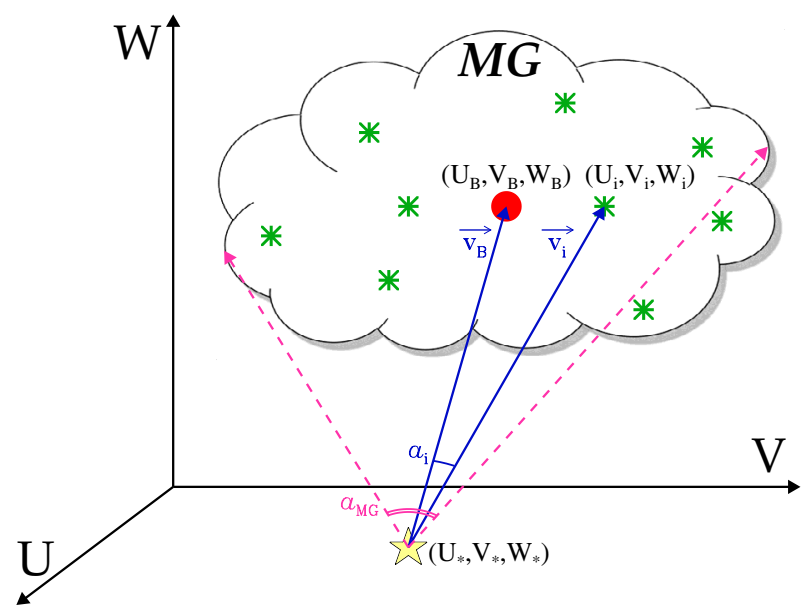

Fig. 2. Illustration of the coordinate system $(v, \alpha)$ and of new parameters defined by the M2M method. The MG (cloud shape), its barycenter (circle), its well known members (asterisks), and the candidate (star symbol) for which the membership is to be tested by our statistical methods are shown.

of this method by splitting the distribution in three 1D plots instead of a unique 3D representation. Figure 1 shows the case of a source with velocity components $\left(U_{\star}, V_{\star}, W_{\star}\right)=(12.1$, $-21.3,-6.3) \mathrm{km} \mathrm{s}^{-1}$ that turns out to be a kinematic candidate of the Pleiades SKG with a high probability $\left(P_{3 \mathrm{D}} \sim 95 \%\right.$ and $\left.P_{2 \mathrm{D}} \sim 98 \%\right)$. Although two of its velocity components are very close to the centers of the distributions for IC 2391 and Castor, the membership probability in these groups is very low.

\subsection{Second method: member-to-member (M2M) analysis}

With this method, we considered the complexity of each SKG more. We studied the membership through the observed velocity distribution of its members in the $(v, \alpha)$ coordinate system (Fig. 2), as defined from the space-velocity components $(U, V, W)$.

We changed the reference system so as to have the coordinates of the barycenter of each $\mathrm{MG}\left(U_{B}, V_{B}, W_{B}\right)$ equal to the values found by the $1 \mathrm{D}$ Gaussian fits (Table 1 ). We call $\boldsymbol{v}_{\mathrm{B}}$ the relative velocity of the barycenter with respect to a candidate member with velocity components $\left(U_{\star}, V_{\star}, W_{\star}\right)$. The $i$ th member of the MG with velocity components $\left(U_{i}, V_{i}, W_{i}\right)$ has a relative velocity $\boldsymbol{v}_{i}$ with respect to the candidate. The angle $\alpha_{i}$, subtended by the velocity vectors $\boldsymbol{v}_{\mathrm{B}}$ and $\boldsymbol{v}_{i}$ (Fig. 2), is considered positive when the rotation is counterclockwise. Finally $\alpha_{\mathrm{MG}}$ corresponds to the largest angle $\left(\alpha_{\mathrm{MG}}=\max \left(\alpha_{i}\right)-\min \left(\alpha_{i}\right)\right)$ that inscribes the whole MG members as seen from the candidate.

In each $\left(\boldsymbol{v}_{\mathrm{B}}, \boldsymbol{v}_{i}\right)$ plane, we can study the proximity of our candidate to both the MG centre and the $i$ th member. This source will have a high chance of being a kinematical member of a given SKG, if it shares the same global motion as the other sources forming the MG. Conceptually, this implies a small modulus for both velocity vectors $\boldsymbol{v}_{\mathrm{B}}$ and $\boldsymbol{v}_{i}$ and a large value for the angle range $\alpha_{\mathrm{MG}}$, up to $\sim 360^{\circ}$. This can be easily understood if we think about putting the candidate star of Fig. 2 inside the "cloud" of all the known members; in this case (high membership probability) this star will be surrounded by many members with random orientations of the vector $\boldsymbol{v}_{i}$, which implies a large range of $\alpha_{i}$. By the contrary, if the values of the modulus of both velocity vectors $\boldsymbol{v}_{\mathrm{B}}$ and $\boldsymbol{v}_{i}$ are very large and the angle $\alpha_{\mathrm{MG}}$ is very small, as shown in Fig. 2, the candidate does not belong to 
A. Klutsch et al.: Reliable probabilistic determination of membership in stellar kinematic groups in the young disk

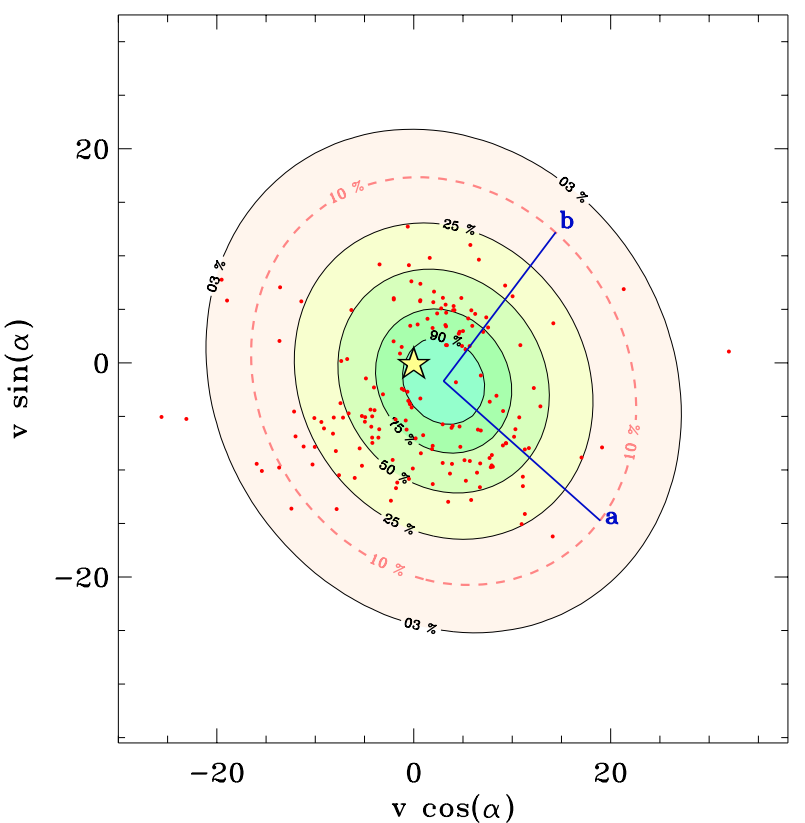

Fig. 3. Candidate locus (star symbol) and known MG members (dots) in the $(v \cos \alpha, v \sin \alpha)$ plane. The probability levels are shown by means of contours (solid lines) derived from the best 2D Gaussian fit. The dashed line represents the membership threshold fixed at $10 \%$. The $(a, b)$ subspace defined by the M2M method is indicated as well.

this SKG. The most critical cases are those corresponding to an intermediate position.

To proceed with our M2M method, we projected all the $\left(\boldsymbol{v}_{\mathrm{B}}, \boldsymbol{v}_{i}\right)$ planes onto a single one (v $\cos \alpha, \mathrm{v} \sin \alpha$; Fig. 3$)$. We thus computed the member density, which was obtained by fitting the whole distribution with a two-dimensional (2D) Gaussian function whose parameters (coordinates of the centroid, orientation of both ellipse's major axis $a$ and minor axis $b$, and density variance $\sigma_{a}$ and $\sigma_{b}$ ) were determined by minimizing the residual. The coordinate center of the $(a, b)$ subspace is the center of the 2D Gaussian that fits the whole distribution and is not coincident with the barycenter of the 3D Gaussian profile (Fig. 2). Finally, we determined its $\left(X^{\prime}, Y^{\prime}\right)$ components in the $(\mathrm{a}, \mathrm{b})$ subspace (Fig. 3) for each candidate. From all these information and in the case of a 2D normal distribution, we computed the membership probability using the following equation:

$P_{2 \mathrm{D}}=\mathrm{e}^{-0.5 k^{2}}$,

where $k=\sqrt{\left(\frac{X^{\prime}}{\sigma_{a}}\right)^{2}+\left(\frac{Y^{\prime}}{\sigma_{b}}\right)^{2}}$ is the normalized velocity modulus of known members to the star candidate in the current coordinate system. The probability level of membership is shown by means of a collection of contours (Fig. 3).

The uncertainty on $P_{2 \mathrm{D}}$ (Fig. A.2) is given by

$\Delta P_{2 \mathrm{D}}=\left|\frac{\partial P_{2 \mathrm{D}}}{\partial k}\right| \Delta k=k \mathrm{e}^{-0.5 k^{2}} \Delta k$.

Considering that the error estimate of $k$ depends only on the uncertainty of the kinematic of the candidate, the change of reference system should not significantly affect its value. We therefore used $\Delta k$ estimated by Eq. (4) to calculate $\Delta P_{2 \mathrm{D}}$.

As before, we fixed the probability cutoff at $10 \%$ (i.e., $k \lesssim$ $2.15)$ to classify a target as a likely SKG member. In addition, the new parameter $\alpha_{\mathrm{MG}}$ is a further criterion for membership in an SKG that has to be used with the probability cutoff of the 2D
Table 2. Membership categories in the M2M method.

\begin{tabular}{cccl}
\hline \hline $\begin{array}{c}\text { Membership group } \\
\text { of candidates }\end{array}$ & $\begin{array}{c}\text { Probability } \\
P_{2 \mathrm{D}}\end{array}$ & $\begin{array}{c}\text { Angle range } \\
\alpha_{\mathrm{MG}}\end{array}$ \\
\hline $\mathrm{G}-$ Good & $\geqslant 10 \%$ & $\geqslant 160^{\circ}$ \\
$\mathrm{P}-$ Possible & $\geqslant 10 \%$ & $130^{\circ}-160^{\circ}$ \\
$\mathrm{U}-$ Unlikely & $\geqslant 10 \%$ & $<130^{\circ}$ \\
$\mathrm{N}-$ Non-member & $<10 \%$ & - \\
\hline
\end{tabular}
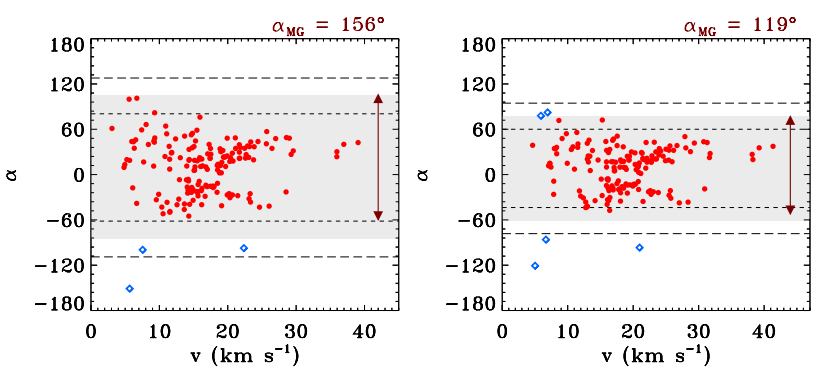

Fig. 4. Distribution of $\alpha$ as a function of $v$ for known members of the Pleiades SKG, as seen from two likely candidates $\left(\alpha_{\mathrm{MG}} \gtrsim 200^{\circ}\right)$ from a first estimate. The gray shading denotes the area of $\pm 4 \sigma$ around the mean angle, while the dashed and long-dashed lines delimit the area if we use a $3 \sigma$ clipping and a $5 \sigma$ clipping, respectively instead. Known members fulfilling this angle criterion are displayed as dots, while the diamonds are used for disregarded ones. The adopted $\alpha_{\mathrm{MG}}$, written at the top of each panel, is shown by an arrow.

Gaussian fit. The Table 2 lists our four membership categories. The angle ranges have been fixed on the basis of geometrical assumptions for getting a good compromise between the angle distribution and any possible scattering along a given direction.

An uneven distribution of the known members in the velocity space affects the $\alpha_{\mathrm{MG}}$ value that may be very large owing to only a few members with discrepant parameters. To avoid this problem, we only selected the SKG members whose angle $\alpha_{i}$ is in the range of $\pm 4 \sigma$ around the mean angle of the distribution. Regarding the typical angle distributions of such cases (Fig. 4), the use of a $4 \sigma$ clipping is more suitable than a $3 \sigma$ clipping (too restrictive) and a $5 \sigma$ clipping (too permissive). We initially classified the two targets of Fig. 4 as good kinematic candidates because their angle $\alpha_{\mathrm{MG}}$ was larger than $200^{\circ}$. After the application of our angular criterion, their final membership category was changed, even drastically. The angle $\alpha_{\mathrm{MG}}$ of the first object is lowered to $156^{\circ}$ (Fig. 4, left panel), which is fairly close to the threshold between "G" and "P" categories. We finally considered it as a likely member of this SKG. Regarding the second case (Fig. 4, right panel), this source has been completely disregarded and demoted within the group of unlikely candidates due to its too small solid angle $\left(\alpha_{\mathrm{MG}} \sim 120^{\circ}\right)$.

\section{Results from Monte Carlo simulations}

The two probabilistic methods to determine the membership in an SKG presented here are based on geometrical properties of the representative points for its members in the kinematical space $(U, V, W)$. The first one is a direct method relating the distance of the candidate to the barycenter with a $3 \mathrm{D}$ normal probability and the second one considers relationships between distances of the candidate to all the known members of the stellar stream, which reduces the problem to a 2D normal distribution with an additional angular criterion. Formally, both methods 
must lead to similar results because they are based on the same geometrical properties of the kinematic space. Nevertheless, because there is no exact equivalence between both methods, we expect that the imposed conditions (i.e., probability cutoff at $10 \%$ ) could lead to different results from each method, principally, in marginal or doubtful cases.

To gain insight into this issue, the use of Monte Carlo simulations (MCSs) helped us to gauge the reliability of one of our techniques with respect to the other (Sect. 3.1). In this way, we can have a better idea of the constraints inherent to each kinematic structure that may bias the interpretation of some results. We then carried out a detailed analysis of the resulting relation of the probabilities $P_{2 \mathrm{D}}$ and $P_{3 \mathrm{D}}$ that are obtained from MCSs (Sect. 3.1.1) and the three kinematic diagrams, $V$ versus $U, W$ versus $V$, and $W$ versus $U$ (Sect. 3.1.2). The comparison of our results with the actual member distribution for each SKG allowed us to understand the role played by the inhomogeneous distribution of members, which is treated properly by the M2M method. In particular, we noted that the M2M method allows us to discover additional possible candidates, which are not identified by the CES method that considers SKGs as being homogeneous kinematical structures.

\subsection{Testing the robustness of our methods}

For each SKG, we constructed two separate samples that are a priori only composed of either members or non-members, respectively. We selected both samples by means of random drawings, which follow a uniform distribution in an area of $\pm 4 \sigma$ (for the three typical spreads in $U, V$, and $W$ coordinates) around the barycenter $\left(U_{0}, V_{0}, W_{0}\right)$ of each distribution, as listed in Table 1 . Such a wide area avoids biases in the velocity space distribution of selected sources, which also includes the most contentious cases. The latter are located around the external ellipsoidal limit that we assumed here as being the isosurface at $k \sim 2.50$ (Sect. 2.1). The fraction of contentious cases within the non-member samples is inversely proportional to the size of the area used to perform our selection. As an example, the use of an area of $\pm 5 \sigma$ (instead of $\pm 4 \sigma$ ) implies that both probabilities $\left(P_{2 \mathrm{D}}\right.$ and $\left.P_{3 \mathrm{D}}\right)$ of almost all non-member candidates are near $0 \%$. Using the membership probability determined with the CES method, one source is considered as a member candidate when its probability $P_{3 \mathrm{D}}$ is larger than $10 \%$. Otherwise, this source has been added to the list of non-members.

Finally, we worked on a set of ten samples (i.e., 5 SKGs $\times$ 2 membership classes) with each one comprising 4000 sources. Table 3 summarizes the results of our MCSs (see Sects. 3.1.1 and 3.1.2 for a more detailed discussion) for the whole analyzed datasets. In the second line of each sample and each stellar stream, we give the fractions of sources identified as members ("G" plus "P" categories) and non-members ("U" plus "N" categories) with the M2M method. This allows us to directly compare the efficiency of the M2M method according to a membership assumption done with the first procedure. These results show that our two procedures are rather similar in all the cases.

With the M2M method, good candidates were classified kinematically for most of the sources (between 75 and 91\%) that are listed in the member datasets. The IC 2391 SKG constitutes an exception (see Sect. 3.1.2 for explanations) because only $58 \%$ of sources have been well-classified. For $13 \%$ of them, we even obtained a membership probability below our membership threshold. The level of agreement between our two procedures rises to over $91 \%$ (79\% for IC $2391 \mathrm{SKG}$ ) when we considered all the possible candidates ("G" plus "P" categories). Their
Table 3. Overall MCS results for our two methods.

\begin{tabular}{|c|c|c|c|c|}
\hline \multirow[t]{2}{*}{ Samples } & \multicolumn{4}{|c|}{ Pleiades MG } \\
\hline & G & $\mathrm{P}$ & $\mathrm{U}$ & $\mathrm{N}$ \\
\hline Member & \multicolumn{2}{|c|}{$92.3 \%$} & $6.5 \%$ & $7.7 \%$ \\
\hline Non-member & \multicolumn{2}{|c|}{$3.4 \%$} & \multicolumn{2}{|c|}{$96.6 \%$} \\
\hline
\end{tabular}

\begin{tabular}{|c|c|c|c|c|}
\hline \multirow[t]{2}{*}{ Samples } & \multicolumn{4}{|c|}{ IC $2391 \mathrm{SC}$} \\
\hline & G & $\mathrm{P}$ & $\mathrm{U}$ & $\mathrm{N}$ \\
\hline Member & \multicolumn{2}{|c|}{$\mathbf{7 8 . 6} \%$} & \multicolumn{2}{|c|}{$21.4 \%$} \\
\hline Non-member & $0.0 \%$ & $\begin{array}{l}0.4 \% \\
\%\end{array}$ & $\begin{array}{r}1.5 \% \\
9\end{array}$ & $\begin{array}{l}98.1 \% \\
\%\end{array}$ \\
\hline
\end{tabular}

\begin{tabular}{|c|c|c|c|c|}
\hline \multirow{2}{*}{ Samples } & \multicolumn{4}{|c|}{ Castor MG } \\
\hline & G & $\mathrm{P}$ & $\mathrm{U}$ & $\mathrm{N}$ \\
\hline Member & \multicolumn{2}{|c|}{$95.1 \%$} & $0.4 \%$ & $4.5 \%$ \\
\hline Non-member & $\begin{array}{r}2.9 \% \\
6\end{array}$ & $\begin{array}{l}3.9 \% \\
6\end{array}$ & 93.2\% & $\begin{array}{l}85.0 \% \\
\%\end{array}$ \\
\hline
\end{tabular}

\begin{tabular}{|c|c|c|}
\hline \multirow[t]{2}{*}{ Samples } & \multicolumn{2}{|c|}{ UMa MG } \\
\hline & G & $\mathrm{N}$ \\
\hline \multirow[t]{2}{*}{ Member } & $90.5 \% \quad 7.6 \%$ & $0.0 \%$ \\
\hline & $98.1 \%$ & $1.9 \%$ \\
\hline \multirow[t]{2}{*}{ Non-member } & $6.3 \%$ & $4.3 \% \quad 82.5 \%$ \\
\hline & $13.2 \%$ & $86.8 \%$ \\
\hline \multirow[t]{2}{*}{ Samples } & \multicolumn{2}{|c|}{ Hyades SC } \\
\hline & G & $\mathrm{N}$ \\
\hline \multirow[t]{2}{*}{ Member } & $79.4 \% \quad 11.8 \%$ & $8.4 \%$ \\
\hline & $91.2 \%$ & $8.8 \%$ \\
\hline Non-member & $9.5 \%$ & $13.6 \% \quad 76.9 \%$ \\
\hline
\end{tabular}

Notes. For each sample and SKG, we list the fraction of sources in each membership group of the M2M method (Table 2) on the first line. The second line matches those of possible ("G" plus "P" categories) and unlikely ("U" plus "N" categories) candidates. Percentages in boldface indicate where our two methods agree. A sample of size 4000 gives margins of error of $1.5 \%$ and $2 \%$ at $95 \%$ and $99 \%$ confidence levels, respectively.

fraction is poorly sensitive to $\alpha_{\mathrm{MG}}$ ranges used. The only relevant difference is a decrease (larger than the error bars) of good candidates that are then classified as likely ones.

We must also keep in mind that almost all the investigated SKGs in the young disk often have some common kinematic properties. Only the UMa stream is clearly separated from the others in the $(U, V)$ plane. It is not unusual that one source may be reported as a candidate of several groups of comoving stars (see, Guillout et al. 2009, 2010). In such cases, the stellar properties (and a coherent sky position for the candidate to MGs) help recognize the best SKG match.

Regarding non-member samples, we got a similar efficiency. More than $76 \%$ of targets were definitively rejected on the basis 

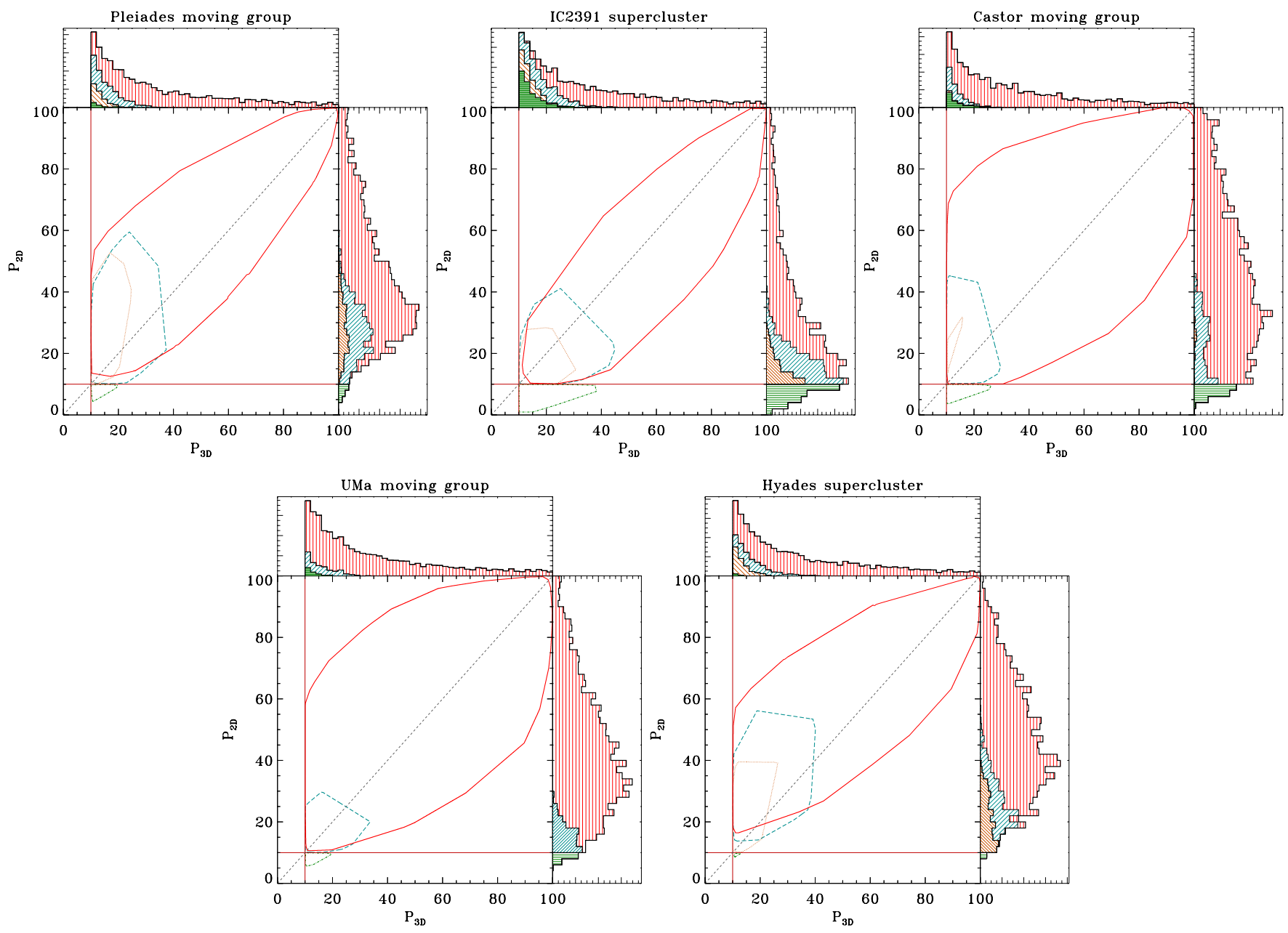

Fig. 5. Comparison of the probability $P_{2 \mathrm{D}}$ relative to $P_{3 \mathrm{D}}$ in the case of the member sample of each MG. On the lower left box of each panel, the solid red, long-dashed blue, dotted orange, and dash-dotted green lines delimit the areas of sources whose classifications from our two methods are in full agreement, possible agreement, possible disagreement, and complete disagreement, respectively. This corresponds to the membership categories "G", "P", "U", and "N" (Table 2). The membership thresholds of both procedures (vertical and horizontal lines) and the one-to-one relation (dashed gray line) are shown as well. In each of the five panels, we plot the histograms of the probabilities $P_{3 \mathrm{D}}\left(\right.$ top box) and $P_{2 \mathrm{D}}($ right box) with a bin size of $2 \%$ using the same color code.

of their kinematics. We exceeded to about $87 \%$ of agreement by including the fraction of unlikely candidates to the total. As before, the results for the IC 2391 SKG stood out because a large fraction of sources has been classified as non-members.

\subsubsection{Relation between the probabilities $P_{2 \mathrm{D}}$ and $P_{3 \mathrm{D}}$}

The distribution of these two probabilities (Figs. 5 and 6, and the more detailed Figs. B.1 to B.5 for each stellar stream and membership category) shows a remarkable scatter of $P_{2 \mathrm{D}}$ for a given $P_{3 \mathrm{D}}$ (and vice versa). We can explain this behavior by the observed inhomogeneity in the known member distribution.

All the sources having a probability $P_{3 \mathrm{D}}$ greater than 30 $45 \%$ (according to the considered SKG) always belong to the "G" group (Fig. 5). When the probability $P_{3 \mathrm{D}}$ reaches the level of $25 \%$, we began to have a mixture of good and possible candidates ("G" and "P" categories), which agrees with our expectations. Some unlikely objects of the IC 2391 supercluster were already found at this stage. By crossing this threshold, we found an inescapable mixture of sources from the four categories.

Regarding the non-member samples (Fig. 6), it is interesting to note that a few sources have a probability $P_{2 \mathrm{D}}$ significantly greater than our threshold. For example, one source of the
UMa non-member sample gets a $P_{2 \mathrm{D}}$ approaching $60 \%$, while its probability $P_{3 \mathrm{D}}$ is slightly lower than $10 \%$. In Sect. 3.2, we look if these kind of sources can also be potential candidates.

\subsubsection{Kinematic distribution}

By analyzing the kinematic distribution of four categories identified with the M2M method in the $(U, V),(V, W)$, and $(U, W)$ planes (Fig. 7 and more detailed Figs. B.6 to B.10 for each SKG and membership category), we found all " $G$ " sources in the middle of each distribution. When adopting ranges of angles wider than those listed in Table 2, the distribution of "G" sources is slightly more concentrated. Close to the probability limits (10\%), some mixing of all the categories occurs. The fraction of other populations increases while that of the best candidates gradually declines, as already pointed in Fig. 5. In some kinematic planes, a stratification of various categories is clearly apparent toward one or more velocity components (i.e., the Hyades MG as shown in Fig. 7), due to some inhomogeneities in the kinematical structure of each SKG.

We already noticed the differences between the observed distributions and their fits. In addition, we are aware to have introduced some biases when considering hypothetical sources with 

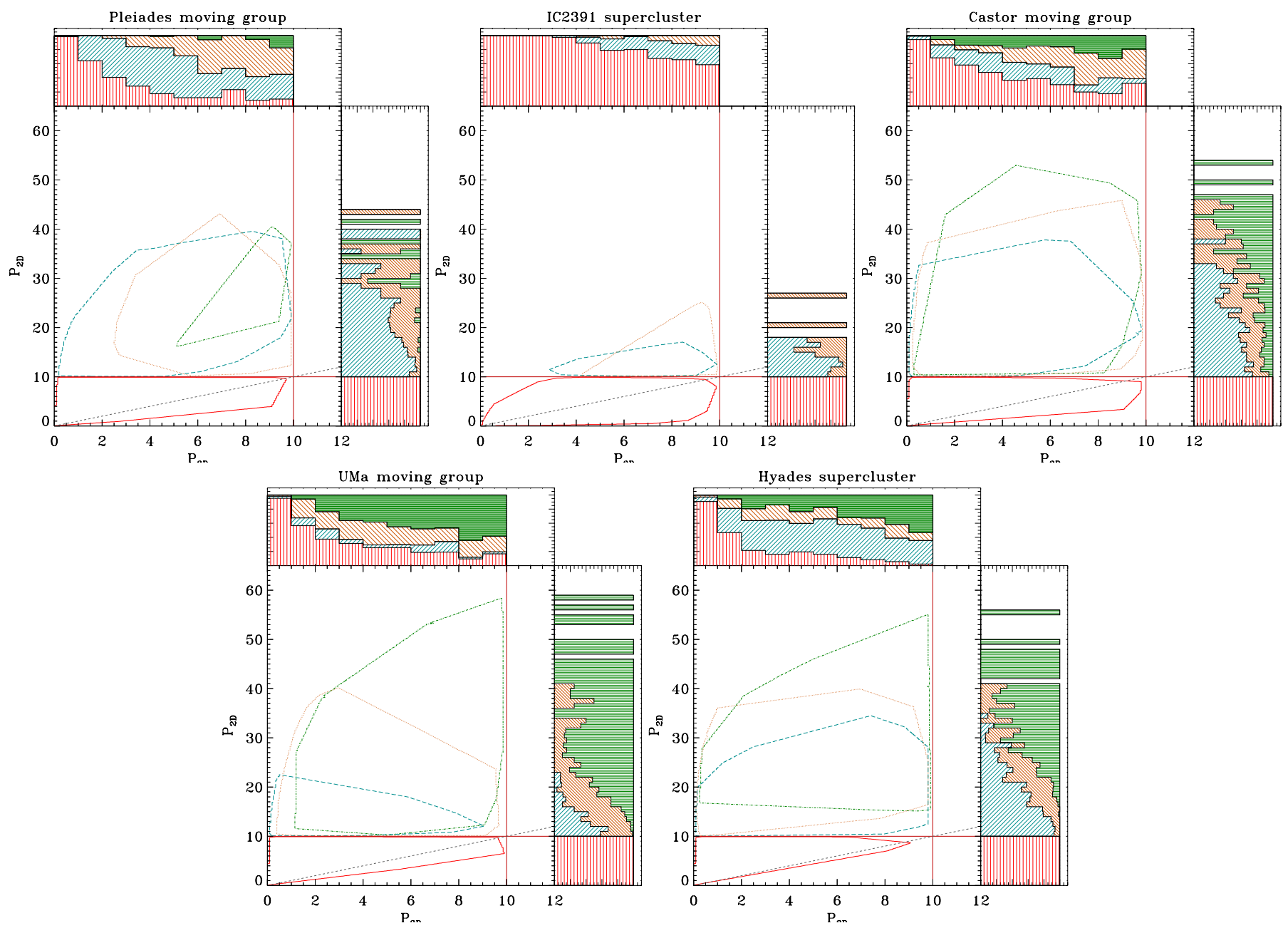

Fig. 6. Comparison of the probability $P_{2 \mathrm{D}}$ relative to $P_{3 \mathrm{D}}$ in the case of the non-member sample of each MG. We used the same line type and color code as in Fig. 5, but these correspond to the membership categories "N", "U", "P", and "G" (Table 2) here. The membership thresholds of both procedures (vertical and horizontal lines) and the one-to-one relation (dashed gray line) are shown as well. We also plot the normalized distribution of the probabilities $P_{3 \mathrm{D}}\left(t o p\right.$ box) and $P_{2 \mathrm{D}}$ (right box), which display the fraction of each category with a bin size of $1 \%$.

kinematics that do not correspond to those of known members of the considered SKG. The best example of this behavior is UMa. For this MG, the distribution of $V$ component is skewed, and the Gaussian fit fails for $V>8 \mathrm{~km} \mathrm{~s}^{-1}$ (Fig. 1). It is therefore not surprisingly that the $V$ component of almost all the misclassified sources is actually in this range. Regarding the other SKGs, most of discrepancies may result from differences between the actual kinematic distribution shown in Fig. 1 and their fit. Such an effect could also arise when we compare our M2M analysis to the others methods that directly compute the likelihood of membership from the distance of the candidate to the barycenter in the velocity space, like the CES method. Hereafter, the discussion refers to the results from the kinematic distribution of the four categories identified with the M2M method in the $(U, V)$, $(V, W)$, and $(U, W)$ planes, as shown in the details of Figs. B.6 to B.10. The comparison with that of the known members listed by Montes et al. (2001) allows us to better understand the intrinsic properties of each SKG.

Pleiades MG: all the sources rejected by the M2M method are located into two specific regions toward the Galactic rotation direction $\left(V<-35 \mathrm{~km} \mathrm{~s}^{-1}\right.$ and $\left.V>-12 \mathrm{~km} \mathrm{~s}^{-1}\right)$, which correspond to the values for which the Gaussian fit is significantly inconsistent with the observed $V$ velocity distribution (Fig. 1). In contrast, these sources have $U$ and $W$ velocities close to the average velocity components in the $(U, W)$ plane. It could be that sources in the high velocity tail of the $V$ distribution are highly probable candidates of the Castor group. We clearly see in the $(U, W)$ and $(V, W)$ planes that the locus of unlikely sources is related to a lack of members in this area. Moreover, this SKG is undoubtedly a more complex structure because several nearby young associations have the same kinematics (Zuckerman \& Song 2004; Torres et al. 2008). As an example, Guillout et al. (2009) found that BD+44 3670 is a highly probable candidate of the Pleiades group, while Zuckerman et al. (2011) proposed it as a Columba association member. A thorough study of the membership for all the Pleiades MG candidates of these young associations is out of the scope of the present paper and will be addressed in a future work.

IC2391 SC: as mentioned before, the results of our two methods are more conflicting for this SKG. A reason could be that only a few members of IC 2391 are reported by Montes et al. (2001) because this supercluster is tightly enclosed between Pleiades and Hyades streams in the velocity space. We can clearly see that the $V$ velocities are concentrated around the 
A. Klutsch et al.: Reliable probabilistic determination of membership in stellar kinematic groups in the young disk
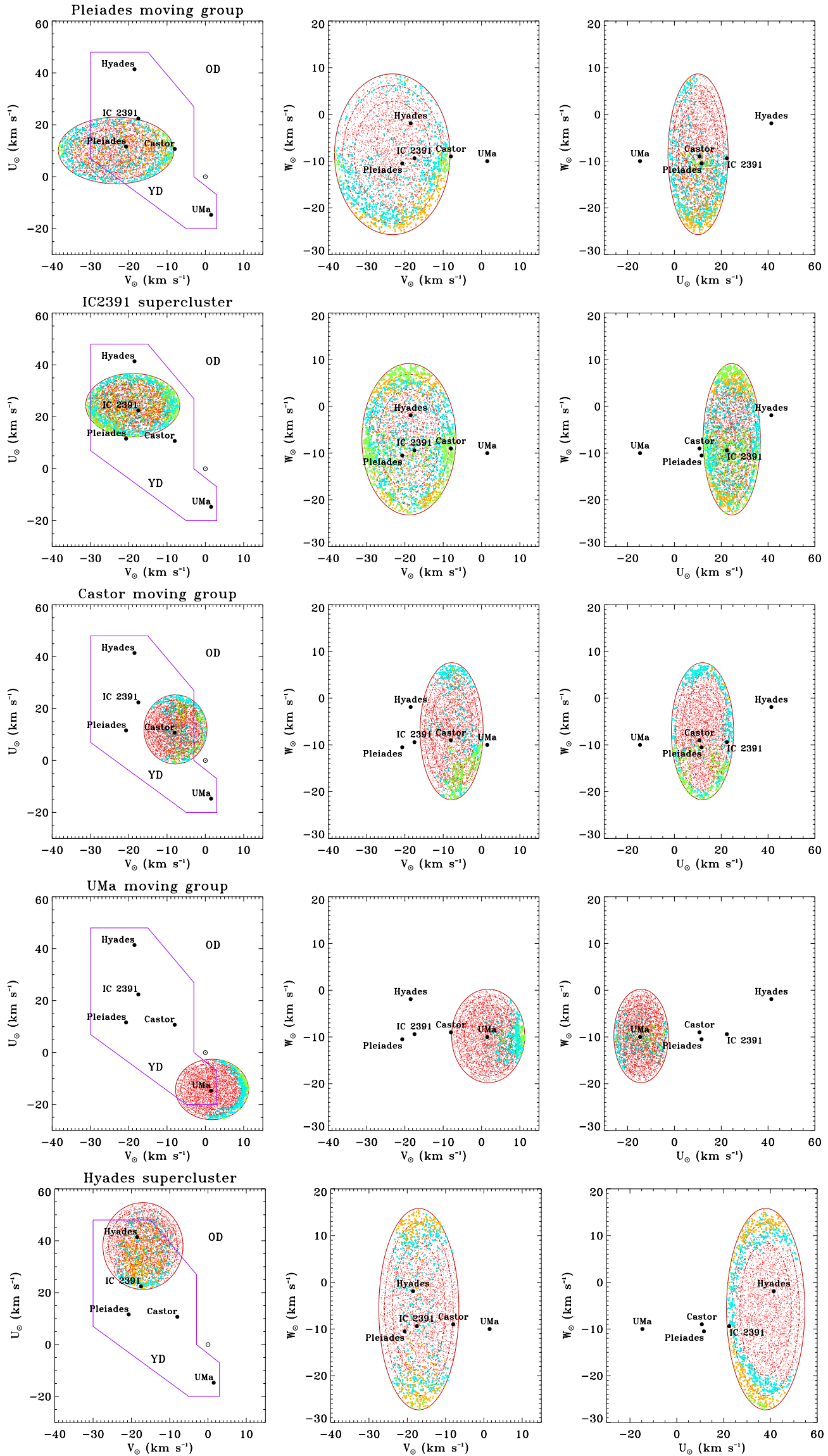

Fig. 7. $(U, V),(V, W)$ and $(U, W)$ planes (from left to right) of sources into the member sample of each studied MGs. We used the same color code as in Fig. 5. The loci of the young-disk (YD) and old-disk (OD) populations (Eggen 1973a,b, 1989) are also marked in the $(U, V)$ plane. The average velocity components of MGs (Montes et al. 2001) are also indicated with filled circles. We also over-plotted elliptical contours of three $k$ values, which correspond to the 10\% threshold for 1D (dashed line), 2D (dash-dotted line), and 3D (solid line) spatial considerations (Fig. A.2). 
mean $V_{0}$, and then its histogram does not have extended wings like the Gaussian profile. In our member sample, we then included a lot of stars that are not compatible within its current velocity boundaries, although the M2M method identifies them. This is completely consistent with results obtained for the nonmember sample where almost all the selected sources are rejected. While there are some members significantly beyond the $10 \%$ threshold in the case of the 2D spatial consideration (dashdotted line in Fig. B.7), all the possible candidates are confined inside this boundary. The effect is stronger in the area of the highest $\mathrm{W}$ velocities because of the large gap in the distribution. With our angle criterion, we discarded the sources farther from the mean of the distribution due to the lack of sources with an intermediate velocity. We notice that the locus of the IC 2391 members corresponds to the area where Bovy et al. (2009) noted the presence of a dearth of stars in the $(U, V)$ plane.

Castor MG: as for the IC 2391 SC, the number of known members is small, but we did not find difficulties in generating our samples to reproduce the observed distribution. The unlikely and rejected sources within the member sample are located into a specific region in the $(V, W)$ plane, which is defined by $V>-12 \mathrm{~km} \mathrm{~s}^{-1}$ and $W<-10 \mathrm{~km} \mathrm{~s}^{-1}$, and actually contains few members.

UMa MG: contrary to the IC $2391 \mathrm{SC}$, this one is well-separated from the others SKGs in all kinematic diagrams, and many members are known. All sources classified as non-members that fulfilled the criteria of the CES method are located where there is a dearth of known members in the $(U, V)$ plane. The majority of them have a velocity $V$ greater than $\sim 8 \mathrm{~km} \mathrm{~s}^{-1}$, which clearly shows the truncation observed in the fit of the distribution toward this direction. We noted that sources listed as possible members are just at current limits in the $(U, V)$ plane and that the large $W$ velocity range is not correctly reproduced.

Hyades SC: the distribution of member candidates by membership categories ("G", "P", "U", and "N") is clearly layered in the $(U, W)$ plane. In the $(V, W)$ plane, this separation is only visible along the $W$ direction, while all classes are more mixed in the $U-V$ diagram. The $W$ velocity of almost all sources classified as good candidates range from -20 to $+10 \mathrm{~km} \mathrm{~s}^{-1}$.

\subsection{Possible members within the non-member sample}

A few non-member sources may also have a high likelihood of kinematic membership when using the M2M method. Out of all of them, we only selected the sources whose probability $P_{3 \mathrm{D}}$ ranges from $7 \%$ to our cutoff at $10 \%$. For a given SKG, we have disregarded those with a higher probability of belonging to another SKG. We divided the remaining sources into three groups according to their probability $\left(P_{2 \mathrm{D}} \geqslant 40 \% ; 30 \% \leqslant P_{2 \mathrm{D}}<40 \%\right.$; $20 \% \leqslant P_{2 \mathrm{D}}<30 \%$ ) with the aim of identifying the impact of the probability estimate on their scattering. Except for IC 2391 SC for the reasons explained previously (see Sect.3.1.2), we discuss their properties within each stellar stream based on their locus on the three kinematic diagrams (Fig. 8).

The analysis of results in the case of the UMa group is very instructive. As its locus in the three Boettlinger diagrams is well separated from those of the other SKGs, none of possible members can be assigned to another SKG. While we started with a uniform distribution, we found that $83 \%$ of sources (34 out of 41) are placed in the young-disk (YD) locus and $85 \%$ of sources (35 out of 41 ) have a particular velocity component toward the galactic rotation direction $\left(V<0 \mathrm{~km} \mathrm{~s}^{-1}\right.$ in Fig. 8). The seven sources with the highest membership probability have some common properties: they are inside the YD population locus, $V$ is close to $-7 \mathrm{~km} \mathrm{~s}^{-1}$ (i.e., near the MG limit toward this direction), and the other components are close to the mean value of the distributions. Regarding the remaining sources, there is no specific distribution in the $(U, W)$ plane.

For the three other streams, the kinematical data are insufficient to draw similar conclusions. In the area of the Pleiades MG, most of these sources have a larger probability of being a member of another SKG. We finally retained only four sources that are also near the IC 2391 group. Potential members to the Castor MG are located in two separate regions toward the Galactic rotation direction. Among the eight sources with a low velocity $\left(V<-10 \mathrm{~km} \mathrm{~s}^{-1}\right)$, only two have a larger probability of membership in this stellar group. The others belong mainly to either the Pleiades or IC 2391 stream. Those in the other area $\left(V>-5 \mathrm{~km} \mathrm{~s}^{-1}\right.$ ) are outside of the current boundary of the YD locus. Finally, for the Hyades group, all the selected sources are close to the external contour in the $(U, V)$ plane. Those with the highest probability are inside or near the locus of YD population. As already seen in the distribution of known members, a sharper selection has taken place toward the north Galactic pole where the $\mathrm{W}$ velocity component is ranging from -20 to $+10 \mathrm{~km} \mathrm{~s}^{-1}$.

Using the M2M method, we are therefore able to push the detection of highly probable members closer to the outer SKGs boundaries, while such sources are unidentifiable by the CES method. Compared to the size of our datasets, these cases are rather marginal because this corresponds to less than $0.2 \%$ of selected sources in the cases of the two young SKGs (i.e., the Pleiades and Castor groups) and about $1 \%$ for the two oldest SKGs (i.e., the UMa and Hyades groups). Nevertheless, we identified G 172-56 as a possible candidate $\left(P_{3 \mathrm{D}}=9.5 \%\right.$ and $P_{2 \mathrm{D}}=30 \%$ ) to the Castor group (see Sect. 4 ).

\section{Application to a stellar sample}

In the previous sections, we worked on test bench sources generated by means of Monte Carlo simulations with only kinematic data. However, the membership of a source in a given stellar group cannot be based only upon the kinematic criteria because it is impossible to separate young and old field stars with a similar space motion, as shown by Bertout \& Genova (2006) for the case of the Taurus-Auriga association. Thus, our statistical methods do not distinguish between coeval stars evaporated from open clusters and other field stars trapped by dynamical processes. Famaey et al. (2007) and López-Santiago et al. (2009) showed that young SKGs are composed by both young and old stars, which, nevertheless, share similar kinematics.

To test the reliability of our methods, we also applied them to a large sample of late-type stars for which both the stellar parameters and kinematics are known. Our choice fell on the dataset of over 160 low-mass stars published by Shkolnik et al. (2009, 2012), for which the stellar age range was well established (Shkolnik et al. 2009, hereafter S09). Shkolnik et al. (2012, hereafter S12) studied their kinematics for identifying the highly probable members of 14 nearby young associations and MGs, using a probabilistic analysis different from ours. That allows us to directly compare their results with those obtained from our statistical methods. Hereafter, our membership index consists of two letters that bring out the results obtained from our two methods. The first letter corresponds to the M2M membership 
A. Klutsch et al.: Reliable probabilistic determination of membership in stellar kinematic groups in the young disk
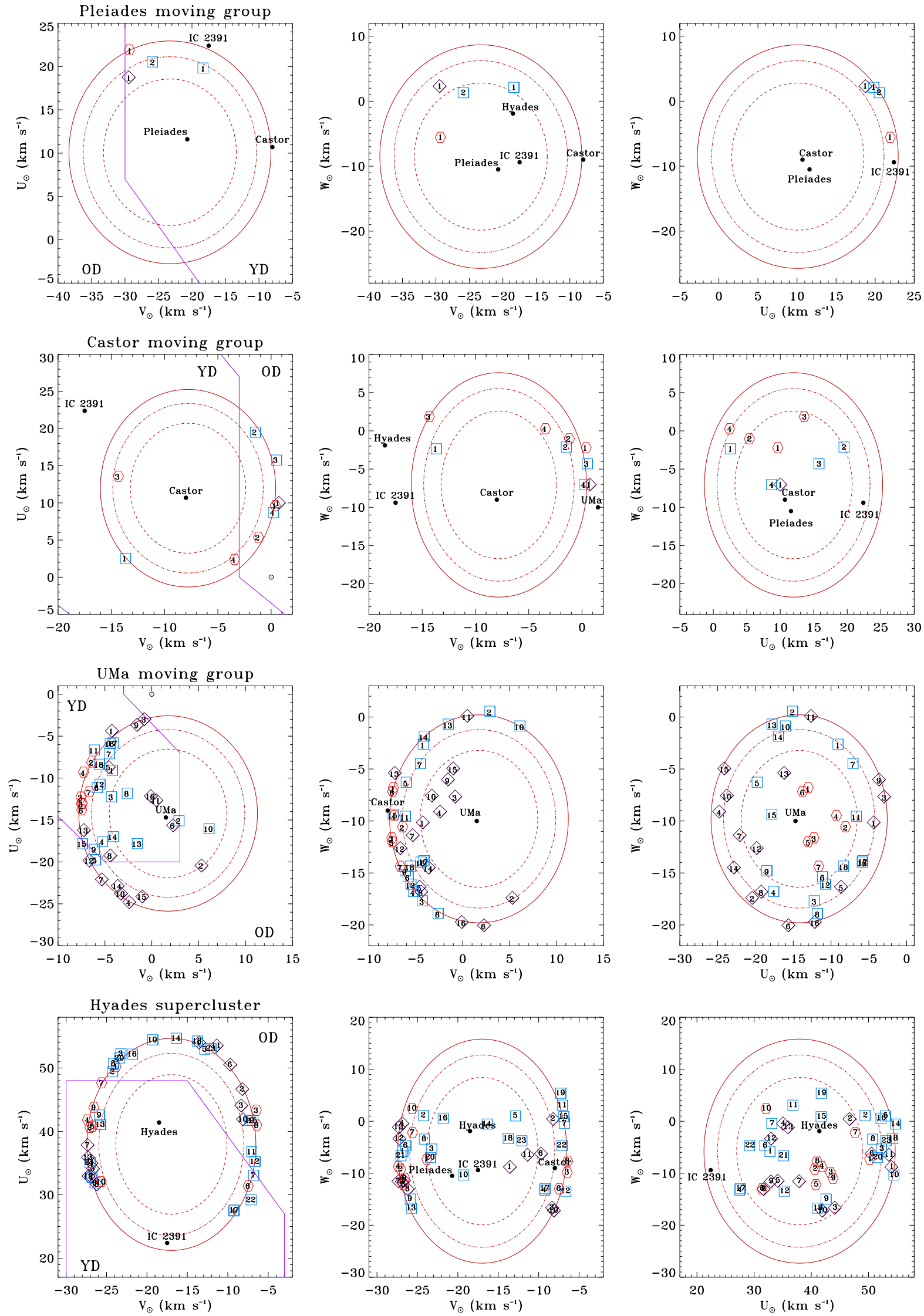

Fig. 8. $(U, V),(V, W)$ and $(U, W)$ planes (from left to right) of all potential candidates $\left(7 \% \leqslant P_{3 \mathrm{D}}<10 \%\right)$ identified into the non-member sample. The YD population and the mean velocities of MGs are indicated as in Fig. 7. We displayed those whose probability $P_{2 \mathrm{D}}$ is larger than or equal to $40 \%$ (hexagons), in the range 30\%-40\% (squares) and 20\%-30\% (diamonds). To visualize the locus of each source in the 3 kinematic diagrams of studied MGs (from top to bottom), we have numbered various symbols. We also over-plotted elliptical contours of three $k$ values, as in Fig. 7. 


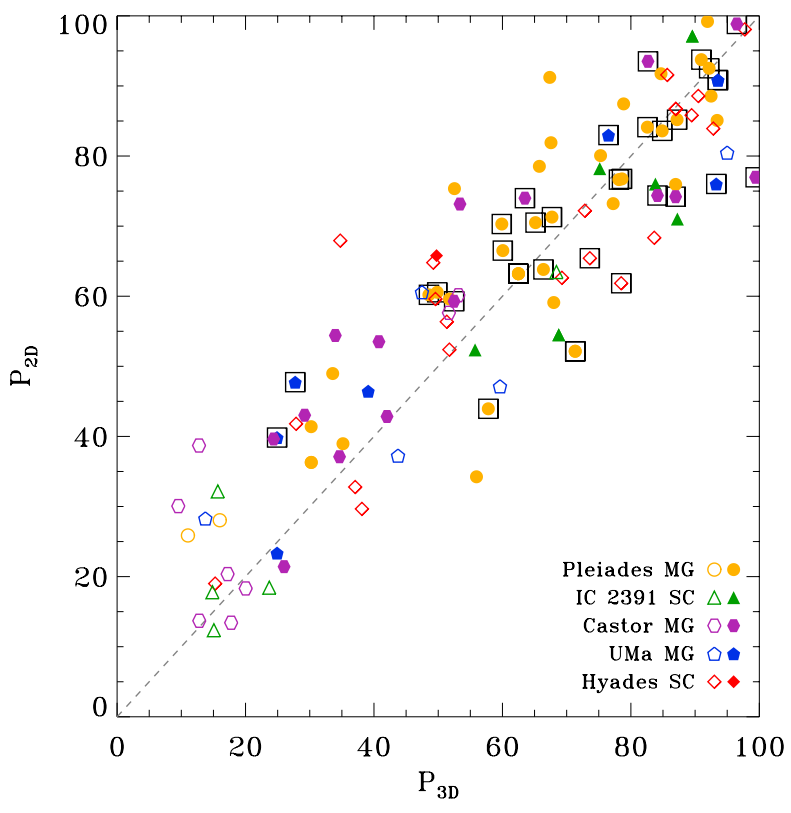

Fig. 9. Comparison of the membership probabilities $P_{2 \mathrm{D}}$ and $P_{3 \mathrm{D}}$ calculated with our methods for S12's low-mass stars. The various symbols are filled when the source is listed as a likely SKG member in Table 4. Otherwise we use open symbols. The highly probable members and possible candidates from S12's work are shown by big squares. The dashed line displays the one-to-one relation.

category as defined in Table 2, while the second one is respectively equal to "Y" or " $N$ " when the candidate is classified as member or non-member from the CES method. We also checked if these sources satisfy the Eggen's criteria on the peculiar and predicted radial velocities using the same cutoffs as Montes et al. (2001). Overall results are listed in Table 4, and the likelihoods of our M2M method versus CES one are plotted in Fig. 9.

We identified 113 sources sharing the same kinematics as one of the SKGs studied in this paper. Whatever the SKG considered, our two probabilities of kinematic membership are similar for all the sources (Fig. 9). The dispersion around the one-toone relation is even smaller than that observed from our MCSs (Fig. 5). Among them, the kinematic match of 105 sources is highly probable with both methods ("GY" quality flag) and six stars are classified as possible members ("PY" quality flag). With kinematic match probabilities $P_{3 \mathrm{D}}=9.5 \%$ and $P_{2 \mathrm{D}}=30 \%$ to the Castor group, G 172-56 is also the unique source of this sample that fulfills the criteria described in the Sect. 3.2. Moreover, as its $\alpha_{\mathrm{MG}}$ is slightly lower than $160^{\circ}$ and its age is estimated to be 60-300 Myr, we cannot discard the possibility that G 172-56 belongs to this SKG. The kinematics of the 54 remaining sources is inconsistent with that of young SKGs investigated here. Most of them are even located outside the area of YD population. For the source G36-26, our methods partially disagree with the S12 analysis. While this source is not associated to none SKG from the CES criteria, we obtained a membership probability $P_{2 \mathrm{D}}$ around $11 \%$, which is slightly larger than our cutoff, to the UMa MG. In none of the cases, we rejected such an association on the basis of our angular criterion (Sect. 2.2).

Almost all the sources identified as highly probable members and possible candidates (i.e., those flagged by S12 as "AAA" and "BAA", respectively) have a high likelihood of membership in one of the SKGs considered by us, which confirms the S12's results (Fig. 9). The only exception is the triple system GJ 1041 (Shkolnik et al. 2010) due to a velocity component toward the north Galactic pole $\left(W \sim 14.70 \mathrm{~km} \mathrm{~s}^{-1}\right)$, which is

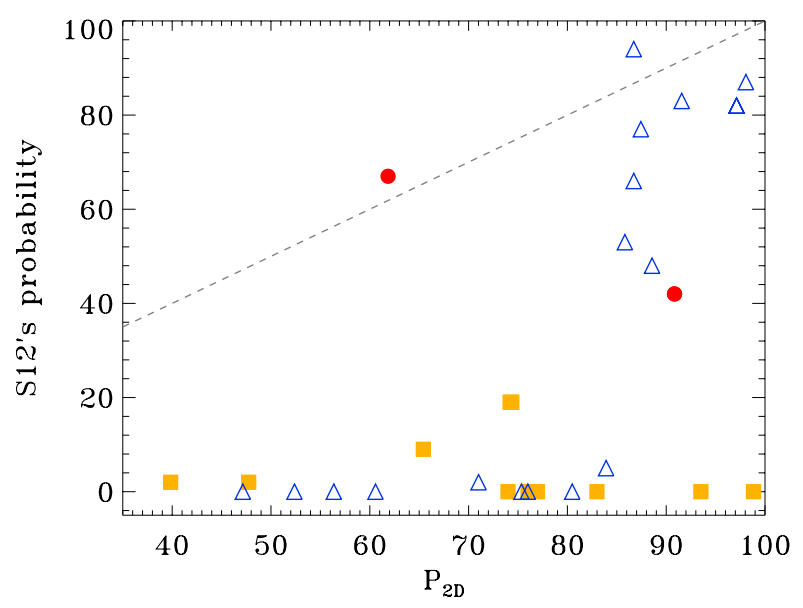

Fig. 10. Comparison of the probability $P_{2 \mathrm{D}}$ relative to that obtained by S12 for the 32 sources with the same best kinematic SKG match from both methods. According to S12's quality flag, we used circles and squares when S12 identified them as likely members and possible candidates, while the triangles indicate the remaining subgroups of sources. The one-to-one relation is displayed by the dashed line.

significantly larger than that of all the tested SKGs. Shkolnik et al. (2012) tentatively proposed this source to the HerculesLyra association. Eisenbeiss et al. (2013) recently revised the member list of this association and found a mean component $W$ around $-8.11 \pm 3.80 \mathrm{~km} \mathrm{~s}^{-1}$. This value is fairly consistent with the range of the velocity component $W$ of the members listed by López-Santiago et al. (2006). Thus, this source does not even belong to the Hercules-Lyra association.

For all the 21 likely members identified by S12, we obtained a kinematic membership probability larger than $40 \%$ with both our methods. Among them, ten sources (G269-153 A, G 269-153 B, G 269-153 C, 1RXS J032230.7+285852, G 80-21, 1RXS J041417.0-090650, CD-35 2722 AB, GJ2060 A, LP 984-91, and LP 984-92) also satisfy the two Eggen's criteria. For LP 984-92, which has no radial velocity listed in S12, we used that of its common-proper motion companion LP 984-91 (Luyten \& Hughes 1980) to apply the Eggen's criteria. This parameter is available for both sources in Torres et al. (2006) and Fernández et al. (2008), and the radial velocity is nearly the same for the two sources. Allen \& Reid (2008) pointed out that GJ 2060 is a probable quadruple system, which is composed of two widely separated equal-mass binaries. Three RV measurements (Torres et al. 2006; Fernández et al. 2008; Shkolnik et al. 2012) are available in the literature (only for the primary) and range from $-30.1 \pm 1.3 \mathrm{~km} \mathrm{~s}^{-1}$ to $-26.6 \pm 1.0 \mathrm{~km} \mathrm{~s}^{-1}$. Using the equations provided by Montes et al. (2001), the Eggen's predicted radial velocity of this system turns out to be $-35.3 \mathrm{~km} \mathrm{~s}^{-1}$. Both Eggen's criteria are only fulfilled when the radial velocity determination done by $\mathrm{S} 12$ is considered.

Among the 14 S12's candidates (excluding GJ 1041 A) with a low match probability but good agreement of their age and sky position (i.e., the "BAA" sources), there are 11 sources for which the best stellar group is one of investigated SKGs (six candidates of the Castor SKG, four of the UMa group, and one of the Hyades stream). While the S12's probabilities of these sources range from 0 to $20 \%$, we obtained likelihoods of membership larger than $60 \%\left(P_{3 \mathrm{D}}>74 \%\right.$ and $\left.P_{2 \mathrm{D}}>63 \%\right)$ from both our methods (Fig. 10). We note that the probability $P_{3 \mathrm{D}}$ of $1 \mathrm{RXS} \mathrm{J} 111300.1+102518$ and G $10-52$ is estimated to about $25 \%$. Regarding the UMa group (Fig. 5), a probability $P_{2 \mathrm{D}}$ larger than $40 \%$ is enough to consider them as good 
A. Klutsch et al.: Reliable probabilistic determination of membership in stellar kinematic groups in the young disk
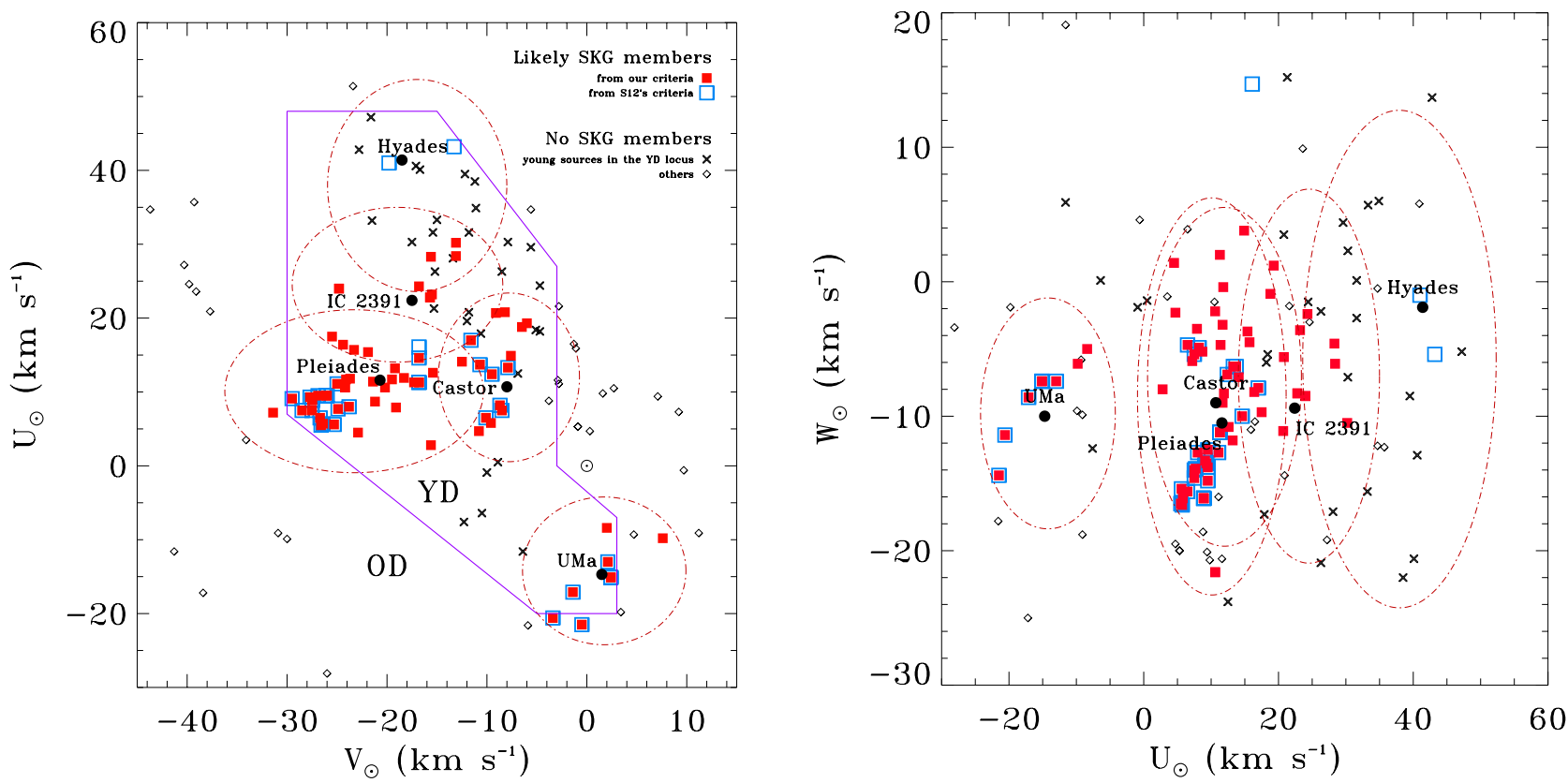

Fig. 11. Distribution of the low-mass stars from Shkolnik et al. (2012) in the $(U, V)$ plane (left panel) and in the $(U, W)$ one (right panel). We show the locus of both the likely SKG members from our methods and those previously identified by S12. The average velocity components of every SKG (Montes et al. 2001) are indicated with filled circles, while the dash-dotted lines correspond to their 20\% threshold in the 3D spatial consideration. In the $(U, V)$ plane, we also over-plotted the loci of the young-disk (YD) and old-disk (OD) populations (Eggen 1973a,b, 1989), which are delimited by the solid line. The position of the Sun is denoted by the corresponding symbol.

kinematic candidates (Fig. 5). Thus, we considered ten of these 11 proposed candidates by $\mathrm{S} 12$ as likely members of their respective SKG. We found that II Tau is a good kinematic candidate of the Hyades stream. Even if this source was already listed as a Hyades candidate by Röser et al. (2011), its membership is still not clear because of the disagreement between its estimated age and that of the Hyades. Due to their very low probability of membership in the AB Doradus association, we proposed GJ 3136 and 1RXS J235133.3+312720 AB as being candidates of the Pleiades MG, mainly because both Eggen's criteria are also fulfilled for this group. The recent discovery of a substellar companion (spectral type of $\mathrm{LO}_{-1}^{+2}$ ) of $1 \mathrm{RXS} \mathrm{J} 235133.3+312720$ (Bowler et al. 2012) seems to provide additional constraints in the possibility of this system belong to the AB Doradus association. As its position is close to the outer limit of the member distributions of the AB Doradus association, the statistical method of S12 (as well as our CES method) cannot reach this source. Only a specific analysis of this distribution following the strategy of our M2M method could confirm this likelihood.

Out of the sources not flagged as "AAA" or "BAA" by S12, we identified 22 possible candidates, which are mostly linked to the Pleiades MG. For 16 of these sources, the properties seem to be more consistent with those of this stellar stream rather that those of the young association listed by S12. With regard to G 271-110 and GJ 3653, we also found that the Pleiades MG is the best kinematic SKG matching with both our methods and Eggen's criteria. The sources NLTT 1875, NLTT 20303 A and B, and 1RXS J193124.2-213422 were already associated to the IC 2391 SC. As we obtained high membership probabilities $(>70 \%)$, these three sources are good candidates of this SKG. With our methods, the sources 1RXSJ073829.3+240014 and G 68-46 seem to belong to the Castor MG instead of the IC 2391 SC and the Chamaeleon-Near MG, as listed by S12, respectively.

We identified 17 more possible candidates for which we obtained a high likelihood of membership and a good age agreement with that of the best SKG match, even if S12 dismissed these stars as non-members (i.e., those with the S12's quality flag "-"). Among them, there are four candidates in the Pleiades MG, four in the IC 2391 SC, six in the Castor MG, two in the UMa MG, and one in the Hyades SC. However, only G 227-22 and 1RXS J022735.8+471021 also satisfy the Eggen's criteria.

A total of 72 sources are identified as likely members and candidates of SKGs, including 39 sources unlisted in the S12's study. We indicate the name of their best SKG match in the last column of Table 4 on the basis of a high likelihood of membership and good age agreement. Figure 11 shows their locus in the $(U, V)$ and $(U, W)$ planes. Most of them (41 sources, i.e., $\sim 57 \%$ of candidates) are located in the region of the Local association, including the 15 and two likely members/candidates that are found by $\mathrm{S} 12$ in the $\mathrm{AB}$ Doradus association and $\beta$ Pictoris $\mathrm{MG}$, respectively. For the remaining SKGs, we found eight additional possible candidates in the Castor MG, eight in the IC 2391 $\mathrm{SC}$, four in the Pleiades MG, two in the UMa MG, and one in the Hyades SC. Taking the S12's members into account, we thus have 14 likely members in the Castor group and eight members each in the IC 2391 and UMa groups.

In addition to 2MASS J04472312-2750358 that is a known Hyades member, we identified 11 highly probable kinematic candidates. The sources II Tau and 1RXS J032230.7+285852 are already listed as candidates of this stream by both $\mathrm{S} 12$ and Röser et al. (2011). While S12 discarded LP 247-13 because of its age, this source was retained by Röser et al. (2011). However, the age quoted by S09 is not consistent with that of the Hyades stream. We found a total of 20 sources that have the same velocity components of the Hyades members. Among them, 11 sources are assigned to this SKG by all the used methods, in spite of their youth and S12's match quality. None of these sources was listed as likely SKG members in Table 4. A new estimation of age is crucial in drawing more robust conclusions about their belonging to this stream. Most of them are located in the upper right corner of Fig. 10. 
Table 5. Radial velocity variable stars among the young sources of the young-disk area, which are not identified as moving group members.

\begin{tabular}{lcl}
\hline \hline Target name & $\begin{array}{c}R V \\
\left(\mathrm{~km} \mathrm{~s}^{-1}\right)\end{array}$ & \multicolumn{1}{c}{ Reference $^{a}$} \\
\hline GJ 3304 $\mathrm{A}^{b}$ & $41.1 \pm 2.6$ & Reid et al. (1995) \\
& $25.5 \pm 1.6$ & Gizis et al. (2002) \\
& $26.7 \pm 1.6$ & Gizis et al. (2002) \\
& $28.1 \pm 1.6$ & Gizis et al. (2002) \\
& $29.8 \pm 1.6$ & Gizis et al. (2002) \\
& $32.87 \pm 0.15$ & Elodie Archive \\
& $32.77 \pm 0.15^{c}$ & Elodie Archive \\
& $35.7 \pm 1.2$ & S12 \\
GJ 3417 A $\mathrm{A}^{d}$ & $26.3 \pm 2.6$ & Reid et al. (1995) \\
& $16.0 \pm 0.1$ & Montes et al. (2001) \\
& $15.0 \pm 0.5$ & Gizis et al. (2002) \\
& $15.4 \pm 0.5$ & Gizis et al. (2002) \\
& $16.1 \pm 0.5$ & Gizis et al. (2002) \\
& $16.86 \pm 0.15$ & Elodie Archive \\
& $16.59 \pm 0.15$ & Elodie Archive \\
& $16.60 \pm 0.15$ & Elodie Archive \\
& $16.43 \pm 0.15$ & Elodie Archive \\
& $16.62 \pm 0.15$ & Elodie Archive \\
& $17.6 \pm 0.6$ & S12 \\
GJ 3730 & $-25.9 \pm 2.6$ & Reid et al. (1995) \\
& $4 \pm 15$ & García López et al. (2000) \\
& $-22.6 \pm 0.1$ & S12 \\
GJ 9652 A & $-42.3 \pm 2.6^{f}$ & Reid et al. (1995) \\
& $-45.0 \pm 11.5$ & Bobylev et al. (2006) \\
& $-88.8 \pm 1.1$ & S12 \\
& $-73.0 \pm 0.8$ & S12 \\
\hline
\end{tabular}

Notes. ${ }^{(a)}$ Link to the Elodie Archive: http://atlas.obs-hp.fr/ elodie/ ${ }^{(b)}$ Previously identified as a visual binary with a separation less than 1 arcsec (Beuzit et al. 2004; Daemgen et al. 2007). ${ }^{(c)}$ During this observations, the cross-correlation function has been fitted with two Gaussian profiles. The radial velocity of potential second component is $47.34 \mathrm{~km} \mathrm{~s}^{-1}$ but with a very small amplitude (only one fifth of the main peak). ${ }^{(d)}$ GJ 3417 A has been identified as a visual binary by S09. (e) Previously listed as SB1 system by S12. GJ 3730 were proposed as candidate of the 500 Myr-old Coma Berenices open cluster by Randich et al. (1996) but later disregarded by García López et al. (2000) due to its location significantly above the $600 \mathrm{Myr}$-isochrone by about $1.5-2 \mathrm{mag}$ in the $V$ vs. $V-I$ and $I$ vs. $R-I$ color-magnitude diagrams. ${ }^{(f)}$ The radial velocity measurement of GJ 9652 B, as provided by Reid et al. (1995), is about $-45.0 \mathrm{~km} \mathrm{~s}^{-1}$.

In the region delimiting the YD population, we identified ten sources younger than $300 \mathrm{Myr}$ that are still associated to none of the SKGs (Fig. 11). That includes the 1-Myr-old M6.0 binary 2MASS J22344161+4041387, which is the youngest and the farthest target analyzed here. This system is also the first low-mass stars potentially associated with the $\mathrm{LkH} \alpha 233$ group located at 325 pc (Allers et al. 2009). Four of these sources (GJ 3304 A, GJ 3417 A, GJ 3730, and GJ 9652 A) could have a variable radial velocity based on the literature values (Table 5). Regarding the remaining sources, only the radial velocity obtained by S12 is available, and we cannot rule out the possibility of any radial velocity variation attributable to binarity. In particular, S12 resolved G 81-34 AB and 2MASS J20003177+5921289 as binaries. The latter was also identified as a binary with a separation of 0.318 arcsec by Janson et al. (2012). The "excess scatter in declination" noted by S12 for 1RXS J194213.0-204547 should result from the presence of a faint background object at about $5.6 \operatorname{arcsec}$ (Chauvin et al. 2010).

\section{Discussion and conclusions}

We have developed two probabilistic methods (Sect. 2) to determine the membership of a star candidate in an SKG. These methods use the geometrical properties in the statistical distributions of their members in the kinematical $(U, V, W)$ space by an approach that is based on Gaussian spatial densities. The CES method (Sect. 2.1) allows us to define a 3D probability of membership using whole space properties, while a 2D probability can be defined through a supplementary angular criterion from the M2M method (Sect. 2.2). The latter allows us to take the inhomogeneity and complexity of each stellar stream into account. We tested the reliability of both methods thanks to Monte Carlo simulations (Sect. 3.1) and the analysis of a large stellar sample whose properties are well known (Sect. 4).

Our Monte Carlo simulations highlight the consistency between the results of the two methods (more than $90 \%$ of all the cases). Using the CES method, a stellar stream is seen as a centrally condensed ellipsoid in the velocity space. The membership probability of a candidate source can then be determined rapidly. This method can give a reliable result if the candidate is located within the clump of stars. Approaching the SKG border, the decrease in the number of members makes the interpretation of the membership more and more difficult, which results in a mixture of possible and unlikely candidates. The M2M method requires a well tabulated knowledge of studied SKGs because the inhomogeneity in the member distribution is taken into account to determine the kinematic membership probability. In the case of the M2M method, the membership category at which each source belongs was established thanks to a second criterion, which used with the $2 \mathrm{D}$ probability of membership. With this method, we can also push the detection of highly probable kinematic members closer to the outer MG boundary, while they are unidentifiable by the CES method because of their proximity to that limit (e.g., G 172-56). Moreover, all the investigated SKGs, except the UMa one, are overlapped with at least one of the other SKGs in the three Boettlinger diagrams. As we simultaneously analyzed the level at which each source belongs to all of them, we should keep in mind that some candidates can be assigned to several streams with a high likelihood.

Applying our methods to a sample of young low-mass stars (Shkolnik et al. 2009, 2012), we identified 113 sources sharing the same kinematics to one of the investigated SKGs. Since the kinematic criteria alone are not sufficient to distinguish the coeval stars evaporated from open clusters and field stars with the same space motion, we assigned a star to a given SKG only if its age is compatible with that of the SKG. We recovered almost all the likely members and possible candidates proposed by Shkolnik et al. (2012). We also found 39 additional highly probable candidates. We finally identified 72 likely members and candidates of SKGs, which correspond to $\sim 64 \%$ of targets. Moreover, 11 additional sources could belong to the Hyades stream because of a good kinematic agreement obtained with both our methods and Eggen's criteria, even if their age is not consistent with such a membership.

To look for new members of close young associations or to identify the members of disrupted open clusters, it is crucial to have complementary information, such as that provided by the analysis of high-resolution spectra, which aims to derive the lithium abundance and $\mathrm{H} \alpha$ luminosity (e.g., Biazzo et al. 2009; Guillout et al. 2009; López-Santiago et al. 2010; Fröhlich et al. 2012) by the detailed differential abundance analysis (e.g., de Silva et al. 2011; Pompéia et al. 2011; Tabernero et al. 2012) 
and by other existing criteria (e.g., Eggen 1995; Shkolnik et al. 2012; Malo et al. 2013; Gagné et al. 2014). Our probabilistic methods are being used by Klutsch et al. (in prep.), who focus on the RasTyc sample of stellar X-ray sources that are connected to the youngest stellar population in the solar neighborhood for identifying further members of these stellar streams.

The post-HIPPARCos era has completely relaunched the issue of the formation and evolution of our Galaxy by the emergence of a new ingredient in the whole models: the stellar kinematic groups or moving groups. One of the main aims (and challenges) of recent modeling is to reproduce the different clumps of co-moving stars in the solar neighborhood by a better knowledge of their origin. One can only speculate on the impact of Gaia on the astronomy in general (see, Eyer et al. 2012) and on this topic in particular.

Acknowledgements. The authors thank the anonymous referee for constructive comments that improved the paper. The financial support was provided by the Universidad Complutense de Madrid (UCM), the Spanish Ministerio de Ciencia e Innovación (MCINN) under grants AYA2011-30147-C03-02, and the Comunidad de Madrid under PRICIT project S2009/ESP-1496 (AstroMadrid). This work was also partially supported by the Italian Ministero dell'Istruzione, Università e Ricerca (MIUR), which is gratefully acknowledged.

\section{References}

Allen, P. R., \& Reid, I. N. 2008, AJ, 135, 2024

Allers, K. N., Liu, M. C., Shkolnik, E., et al. 2009, ApJ, 697, 824

Antoja, T., Valenzuela, O., Pichardo, B., et al. 2009, ApJ, 700, L78

Asiain, R., Figueras, F., Torra, J., \& Chen, B. 1999, A\&A, 341, 427

Bertout, C., \& Genova, F. 2006, A\&A, 460, 499

Beuzit, J.-L., Ségransan, D., Forveille, T., et al. 2004, A\&A, 425, 997

Biazzo, K., Frasca, A., Marilli, E., et al. 2009, A\&A, 499, 579

Bobylev, V. V., Goncharov, G. A., \& Bajkova, A. T. 2006, Astron. Rep., 50, 733

Bovy, J., \& Hogg, D. W. 2010, ApJ, 717, 617

Bovy, J., Hogg, D. W., \& Roweis, S. T. 2009, ApJ, 700, 1794

Bowler, B. P., Liu, M. C., Shkolnik, E. L., et al. 2012, ApJ, 753, 142

Chakrabarty, D. 2007, A\&A, 467, 145

Chauvin, G., Lagrange, A.-M., Bonavita, M., et al. 2010, A\&A, 509, A52

Chereul, E., Crézé, M., \& Bienaymé, O. 1998, A\&A, 340, 384

Chereul, E., Crézé, M., \& Bienaymé, O. 1999, A\&AS, 135, 5

Daemgen, S., Siegler, N., Reid, I. N., \& Close, L. M. 2007, ApJ, 654, 558

de Silva, G. M., Freeman, K. C., Bland-Hawthorn, J., et al. 2011, MNRAS, 415, 563

De Simone, R., Wu, X., \& Tremaine, S. 2004, MNRAS, 350, 627

Dehnen, W. 1998, AJ, 115, 2384

Eggen, O. J. 1958, MNRAS, 118, 65

Eggen, O. J. 1973a, PASP, 85, 289

Eggen, O. J. 1973b, PASP, 85, 379

Eggen, O. J. 1989, PASP, 101, 366

Eggen, O. J. 1994, AJ, 107, 594

Eggen, O. J. 1995, AJ, 110, 2862

Eggen, O. J. 1996, AJ, 112, 1595

Eggen, O. J., \& Iben, Jr., I. 1988, AJ, 96, 635

Eisenbeiss, T., Ammler-von Eiff, M., Roell, T., et al. 2013, A\&A, 556, A53

Eyer, L., Palaversa, L., Mowlavi, N., et al. 2012, Ap\&SS, 341, 207

Famaey, B., Jorissen, A., Luri, X., et al. 2005, A\&A, 430, 165

Famaey, B., Pont, F., Luri, X., et al. 2007, A\&A, 461, 957

Famaey, B., Siebert, A., \& Jorissen, A. 2008, A\&A, 483, 453

Feltzing, S., \& Holmberg, J. 2000, A\&A, 357, 153
Fernández, D., Figueras, F., \& Torra, J. 2008, A\&A, 480, 735

Fröhlich, H.-E., Frasca, A., Catanzaro, G., et al. 2012, A\&A, 543, A146

Gagné, J., Lafrenière, D., Doyon, R., Malo, L., \& Artigau, É. 2014, ApJ, 783, 121

García López, R. J., Randich, S., Zapatero Osorio, M. R., \& Pallavicini, R. 2000, A\&A, 363, 958

Gizis, J. E., Reid, I. N., \& Hawley, S. L. 2002, AJ, 123, 3356

Griffin, R. F. 1998, The Observatory, 118, 223

Guillout, P., Klutsch, A., Frasca, A., et al. 2009, A\&A, 504, 829

Guillout, P., Frasca, A., Klutsch, A., Marilli, E., \& Montes, D. 2010, A\&A, 520, A94

Janson, M., Hormuth, F., Bergfors, C., et al. 2012, ApJ, 754, 44

Klutsch, A. 2008, Ph.D. thesis, Observatoire de Strasbourg, Universié Louis Pasteur, France

Klutsch, A., Guillout, P., Frasca, A., et al. 2011a, in Highlights of Spanish Astrophysics VI, eds. M. R. Zapatero Osorio, J. Gorgas, J. Maíz Apellániz, J. R. Pardo, \& A. Gil de Paz, 534

Klutsch, A., Montes, D., Guillout, P., et al. 2011b, in 16th Cambridge Workshop on Cool Stars, Stellar Systems, and the Sun, eds. C. Johns-Krull, M. K. Browning, \& A. A. West, ASP Conf. Ser., 448, 661

Klutsch, A., Montes, D., Guillout, P., et al. 2012, in SF2A-2012: Proc. of the Annual meeting of the French Society of Astronomy and Astrophysics, eds. S. Boissier, P. de Laverny, N. Nardetto, et al., 361

López-Santiago, J., Montes, D., Crespo-Chacón, I., \& Fernández-Figueroa, M. J. 2006, ApJ, 643, 1160

López-Santiago, J., Micela, G., \& Montes, D. 2009, A\&A, 499, 129

López-Santiago, J., Montes, D., Gálvez-Ortiz, M. C., et al. 2010, A\&A, 514, A97

Luyten, W. J., \& Hughes, H. S. 1980, Proper Motion Survey with the Forty- Eight Inch Schmidt Telescope, Vol. LV, first supplement to the NLTT Catalogue (Minneapolis: University of Minnesota), 1

Mädler, J. H. 1846, Astron. Nachr., 24, 213

Malo, L., Doyon, R., Lafrenière, D., et al. 2013, ApJ, 762, 88

McMillan, P. J. 2013, MNRAS, 430, 3276

Minchev, I., \& Famaey, B. 2010, ApJ, 722, 112

Minchev, I., \& Quillen, A. C. 2007, MNRAS, 377, 1163

Minchev, I., Boily, C., Siebert, A., \& Bienaymé, O. 2010, MNRAS, 407, 2122

Montes, D., López-Santiago, J., Gálvez, M. C., et al. 2001, MNRAS, 328, 45

Pelat, D. 2006, in Bases et méthodes pour le traitement des données, Master's degree 2nd year handbook (Université de Strasbourg, France)

Pompéia, L., Masseron, T., Famaey, B., et al. 2011, MNRAS, 415, 1138

Proctor, R. A. 1869, Roy. Soc. London Proc. Ser. I, 18, 169

Quillen, A. C. 2003, AJ, 125, 785

Quillen, A. C., \& Minchev, I. 2005, AJ, 130, 576

Randich, S., Schmitt, J. H. M. M., \& Prosser, C. 1996, A\&A, 313, 815

Reid, I. N., Hawley, S. L., \& Gizis, J. E. 1995, AJ, 110, 1838

Röser, S., Schilbach, E., Piskunov, A. E., Kharchenko, N. V., \& Scholz, R.-D. 2011, A\&A, 531, A92

Sellwood, J. A. 2010, MNRAS, 409, 145

Sellwood, J. A. 2011, MNRAS, 410, 1637

Shkolnik, E. L., Liu, M. C., \& Reid, I. N. 2009, ApJ, 699, 649

Shkolnik, E. L., Hebb, L., Liu, M. C., Reid, I. N., \& Collier Cameron, A. 2010, ApJ, 716, 1522

Shkolnik, E. L., Anglada-Escudé, G., Liu, M. C., et al. 2012, ApJ, 758, 56

Skuljan, J., Hearnshaw, J. B., \& Cottrell, P. L. 1999, MNRAS, 308, 731

Tabernero, H. M., Montes, D., \& González Hernández, J. I. 2012, A\&A, 547, A13

Taylor, B. J. 2000, A\&A, 362, 563

Torra, J., Fernández, D., \& Figueras, F. 2000, A\&A, 359, 82

Torres, C. A. O., Quast, G. R., da Silva, L., et al. 2006, A\&A, 460, 695

Torres, C. A. O., Quast, G. R., Melo, C. H. F., \& Sterzik, M. F. 2008, in Handbook of Star Forming Regions, Volume II, ed. B. Reipurth, 5, 757

Zuckerman, B., \& Song, I. 2004, ARA\&A, 42, 685

Zuckerman, B., Rhee, J. H., Song, I., \& Bessell, M. S. 2011, ApJ, 732, 61

Pages 16 to 30 are available in the electronic edition of the journal at http://www . aanda. org 


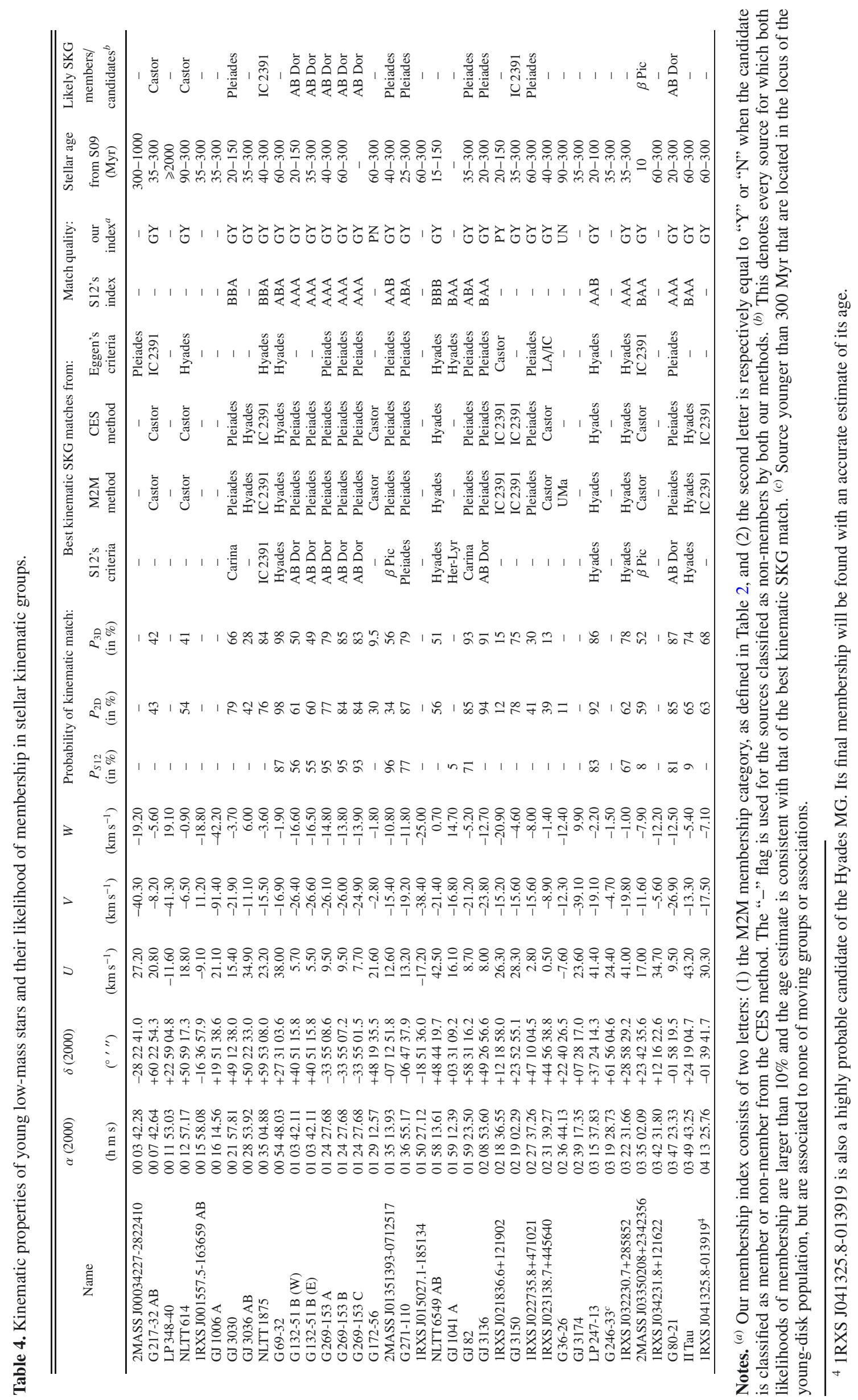


A. Klutsch et al.: Reliable probabilistic determination of membership in stellar kinematic groups in the young disk

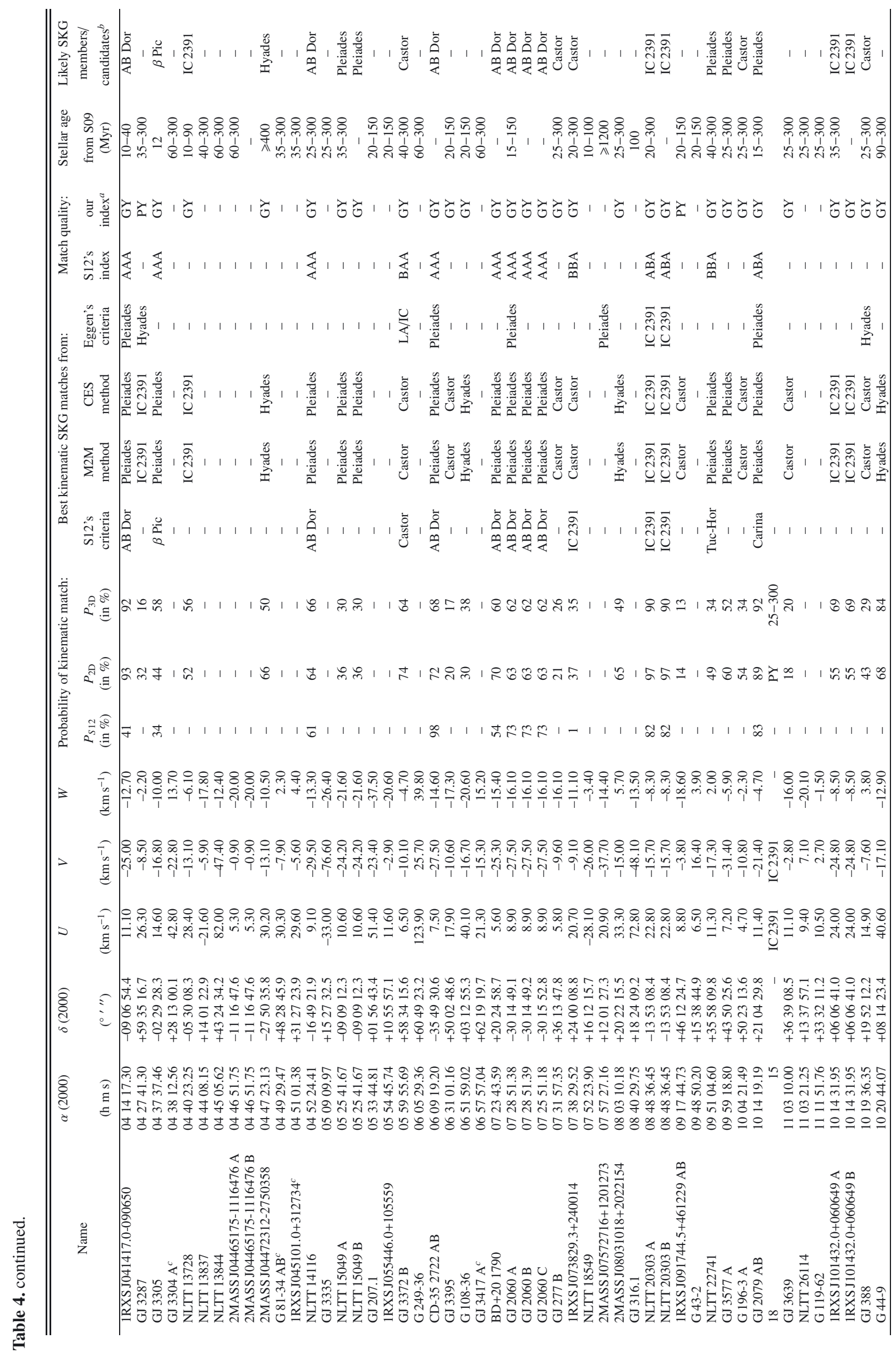

A52, page 17 of 30 


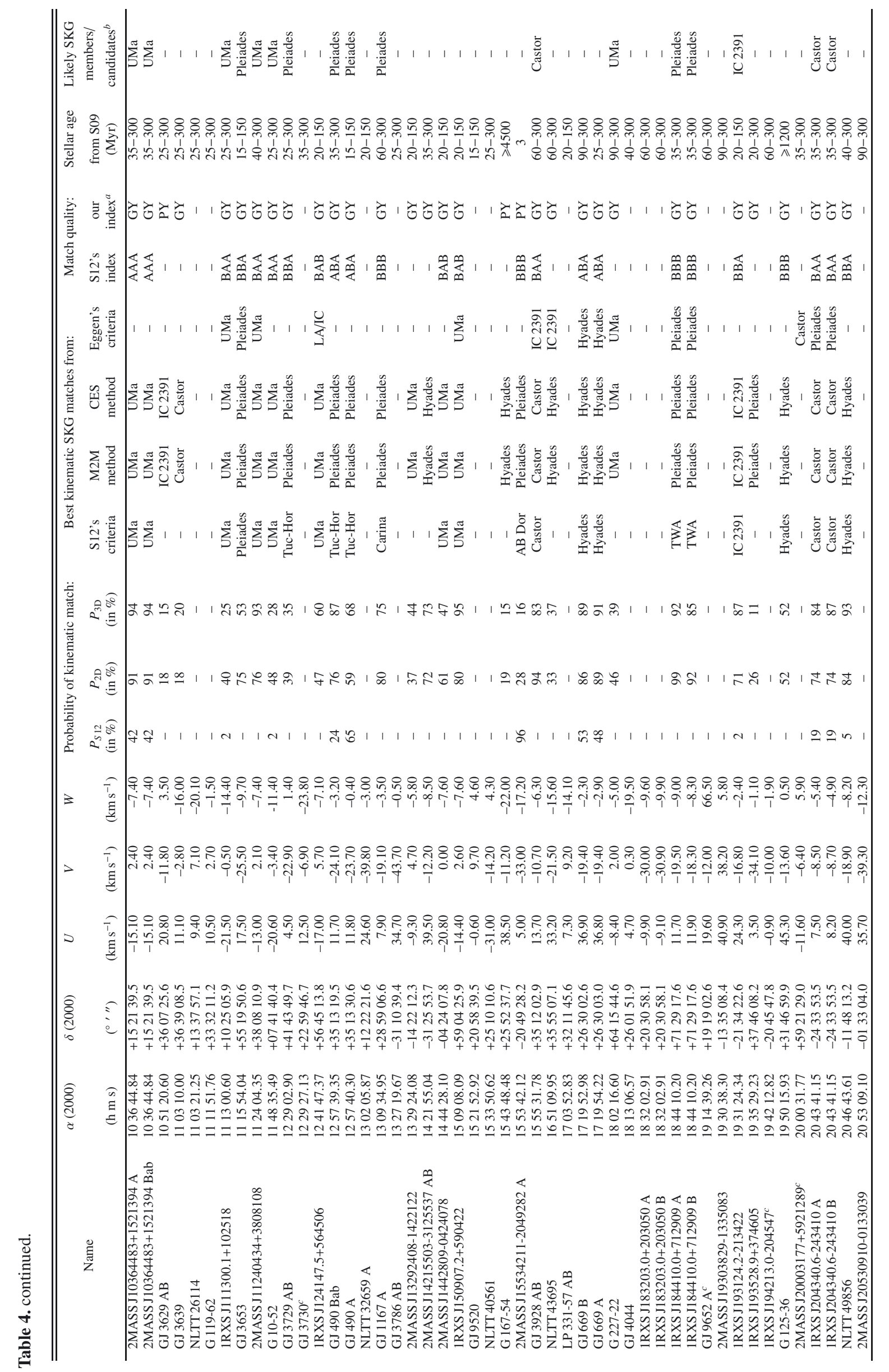


A. Klutsch et al.: Reliable probabilistic determination of membership in stellar kinematic groups in the young disk

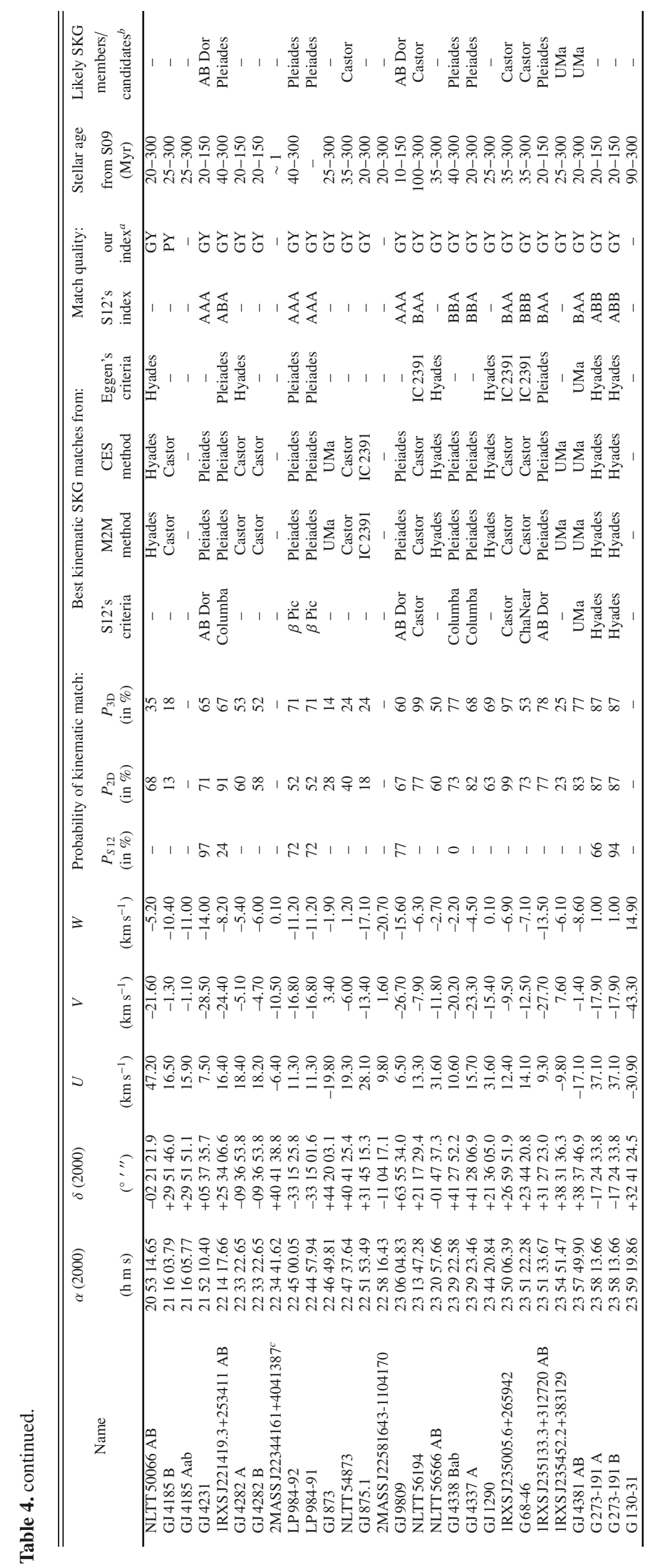




\section{Appendix A: Mathematical tools}

In probability theory, real-valued random variables that tend to cluster around a single mean value are often described by the normal distribution (e.g., a continuous probability distribution) in a first approximation. This method was published by de Moivre $(1738)^{2}$ and was popularized by Gauss $(1809)^{3}$. The associated probability density function $\phi(x)$ of a random variable $x$ following a normal law is given by

$\phi(x)=\frac{1}{\sqrt{2 \pi \sigma^{2}}} \mathrm{e}^{-\frac{(x-\mu)^{2}}{2 \sigma^{2}}}$,

where the parameters $\mu$ and $\sigma^{2}$ are the mean and the variance of the distribution that is usually denoted by $\mathcal{N}\left(\mu, \sigma^{2}\right)$. When this function has a mean zero and unit variance, this is called the standard normal distribution that is denoted $\mathcal{N}(0,1)$. Its associated probability density function (solid line of Fig. A.1) is $\phi(x)=\frac{1}{\sqrt{2 \pi}} \mathrm{e}^{-x^{2} / 2}$ in which the constant $1 / \sqrt{2 \pi}$ ensures that the total area under the curve $\phi(x)$ is equal to one.

The cumulative distribution function (cdf) describes probabilities for a random variable to fall in the intervals of the form $(-\infty, x]$. The cdf of the standard normal distribution, which is denoted $\Phi(x)$, can be computed as an integral of $\phi(x)$ :

$\Phi(x)=\frac{1}{\sqrt{2 \pi}} \int_{-\infty}^{x} \mathrm{e}^{-t^{2} / 2} \mathrm{~d} t=\frac{1}{2}\left[1+\operatorname{erf}\left(\frac{x}{\sqrt{2}}\right)\right]$.

where erf is the error function.

The probability content $P_{k^{2}}$ describes the probability for a random variable $\mathrm{X}$ to fall into the interval from $-k$ to $k$ (Fig A.1). The parameter $k=\frac{X-\mu}{\sigma}$ referees the separation from the center of the distribution related to the standard normal distribution. The analytic expression is given by Pelat (2006) for the $n$-dimensional normal distributions until the third dimension:

- 1D: $\quad P_{k^{2}}=\Phi(k)-\Phi(-k)$,

- 2D: $\quad P_{k^{2}}=1-\mathrm{e}^{-0.5 k^{2}}$,

- 3D: $\quad P_{k^{2}}=2 \Phi(k)-1-\sqrt{\frac{2}{\pi}} k \mathrm{e}^{-0.5 k^{2}}$.

We defined our membership probability (Fig. A.2) as being the complement of the probability content $R(k)=1-P_{k^{2}}$ (i.e., the residual error function in the probability theory). For the onedimensional Gaussian profile, this function gives the probability for a normal random variable to deviate from its mean of $k$ times the standard deviation $\sigma$. When we described our methods (Sect. 2.1 and 2.2), we explicitly gave the formula of the parameter $k$ used during this work.

\footnotetext{
2 In the second edition of The Doctrine of Chances (1738, pp. 235-243). De Moivre first presented privately a brief paper entitled "Approximatio ad Summam Terminorum Binomii $(a+b)^{n}$ in Seriem Expansi" in 1733. This original pamphlet was reprinted several times, as in Walker, H. M. (1985): "De Moivre on the law of normal probability", in A source book in mathematics, by D.E. Smith (Dover), ISBN 0486646904. http://www. york.ac.uk/depts/maths/histstat/ demoivre.pdf

3 In the monograph "Theoria motvs corporvm coelestivm in sectionibvs conicis Solem ambientivm" (1809, Hamburgi: Sumtibus F. Perthes et I.H. Besser original title in Latin) [Theory of the motion of the heavenly bodies moving about the Sun in conic sections] (English translation).
}

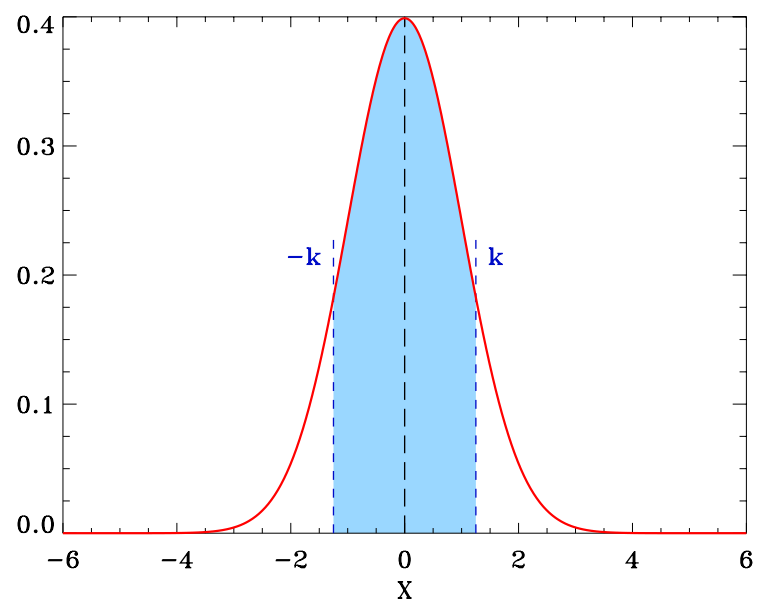

Fig. A.1. Standard normal distribution $\mathcal{N}(0,1)$ (solid red line). The blue-shaded area denotes the probability content for a random variable $X$ of falling into the interval from $-k$ to $k$.

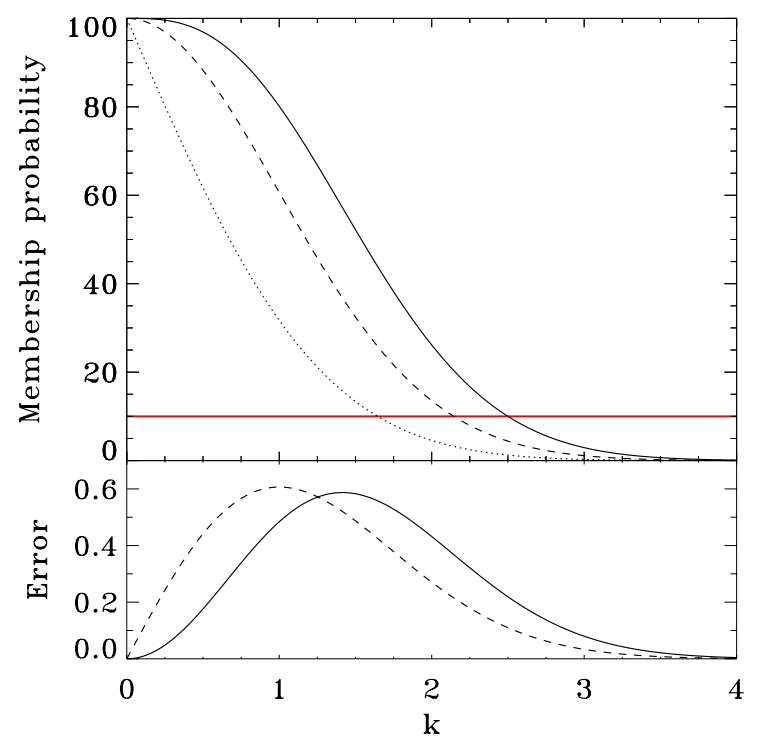

Fig. A.2. Top panel: membership probability of the standard normal random variable $k$ (in unit of the variance) in the case of the standard 1D (dotted line), 2D (Eq. (5); dashed line), and 3D (Eq. (2); solid line) normal distributions. The horizontal line represents the threshold at $10 \%$ used in this work. Bottom panel: associated uncertainty of the membership probability relative to the error estimate of $\mathrm{k}, \Delta k$. This corresponds to the partial derivative of the membership probability with respect to $k$ for the 2D (Eq. (6); dashed line) and 3D (Eq. (3); solid line) normal distributions. 
A. Klutsch et al.: Reliable probabilistic determination of membership in stellar kinematic groups in the young disk

\section{Appendix B}
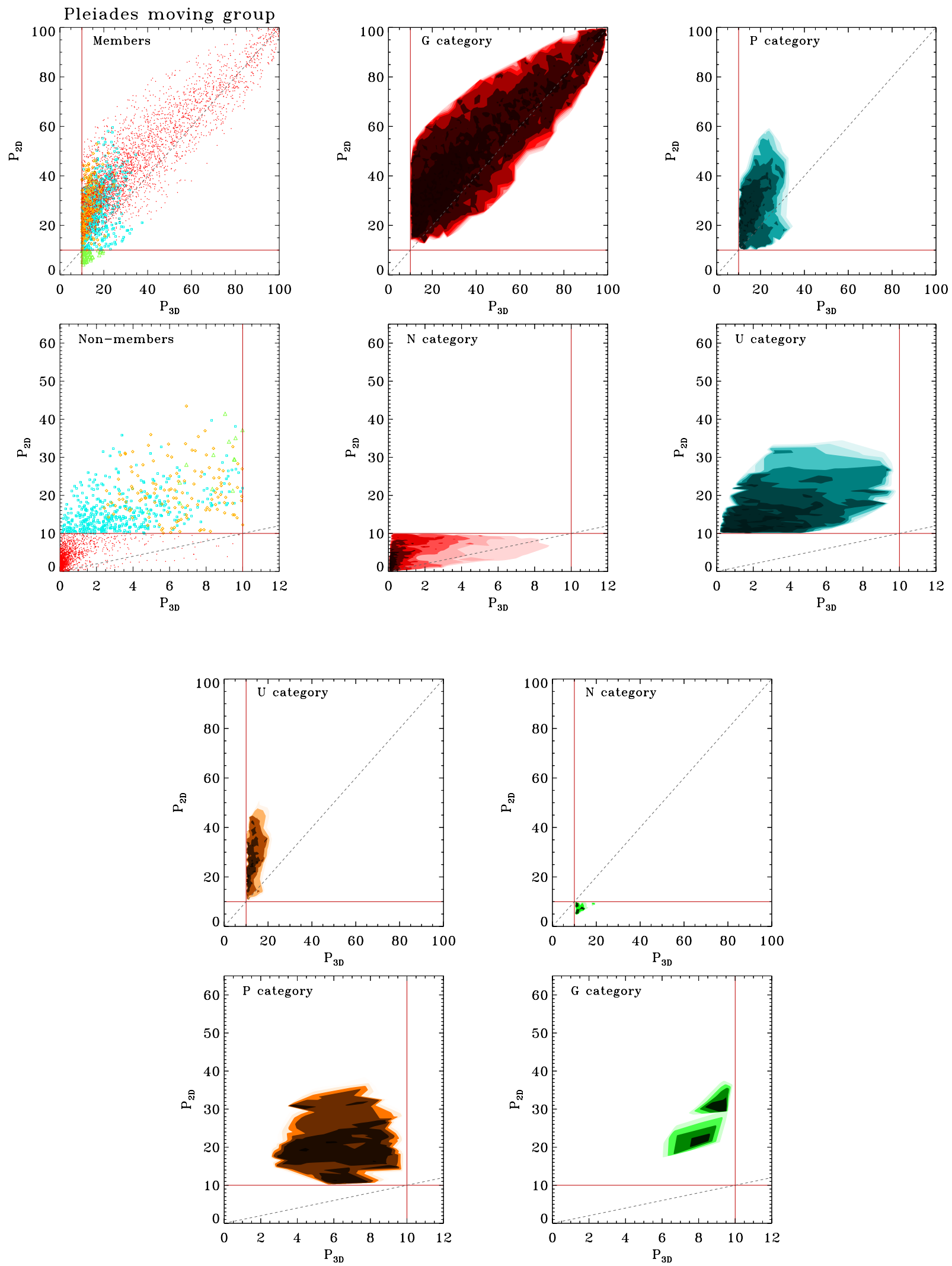

Fig. B.1. Comparison of the $2 \mathrm{D}$ probability $P_{2 \mathrm{D}}$ relative to the $3 \mathrm{D}$ probability $P_{3 \mathrm{D}}$ of the sources that are selected by means of Monte Carlo simulations in the case of the Pleiades MG. Each panel is composed of two plots that show the results for the member (upper plot) and nonmember (lower plot) samples. On the top left panel, we display the distribution of sources whose classifications from both of our methods are in full agreement (red), possible agreement (blue), possible disagreement (orange), and complete disagreement (green). On the other panels, we present such a distribution for every membership status where the color scale codes the density gradient. 
A\&A 567, A52 (2014)
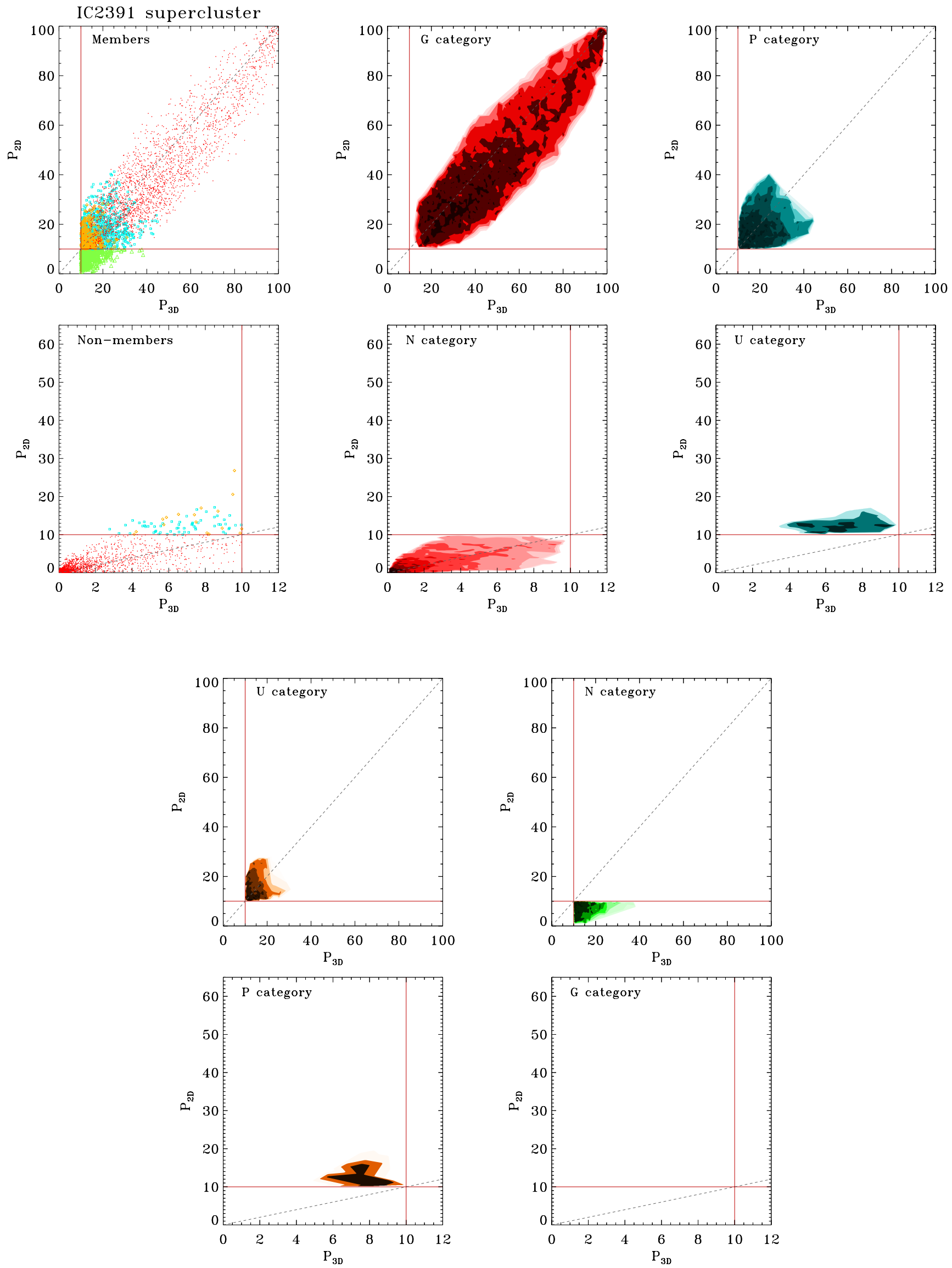

Fig. B.2. As Fig. B.1 but for the IC 2391 supercluster. 
A. Klutsch et al.: Reliable probabilistic determination of membership in stellar kinematic groups in the young disk
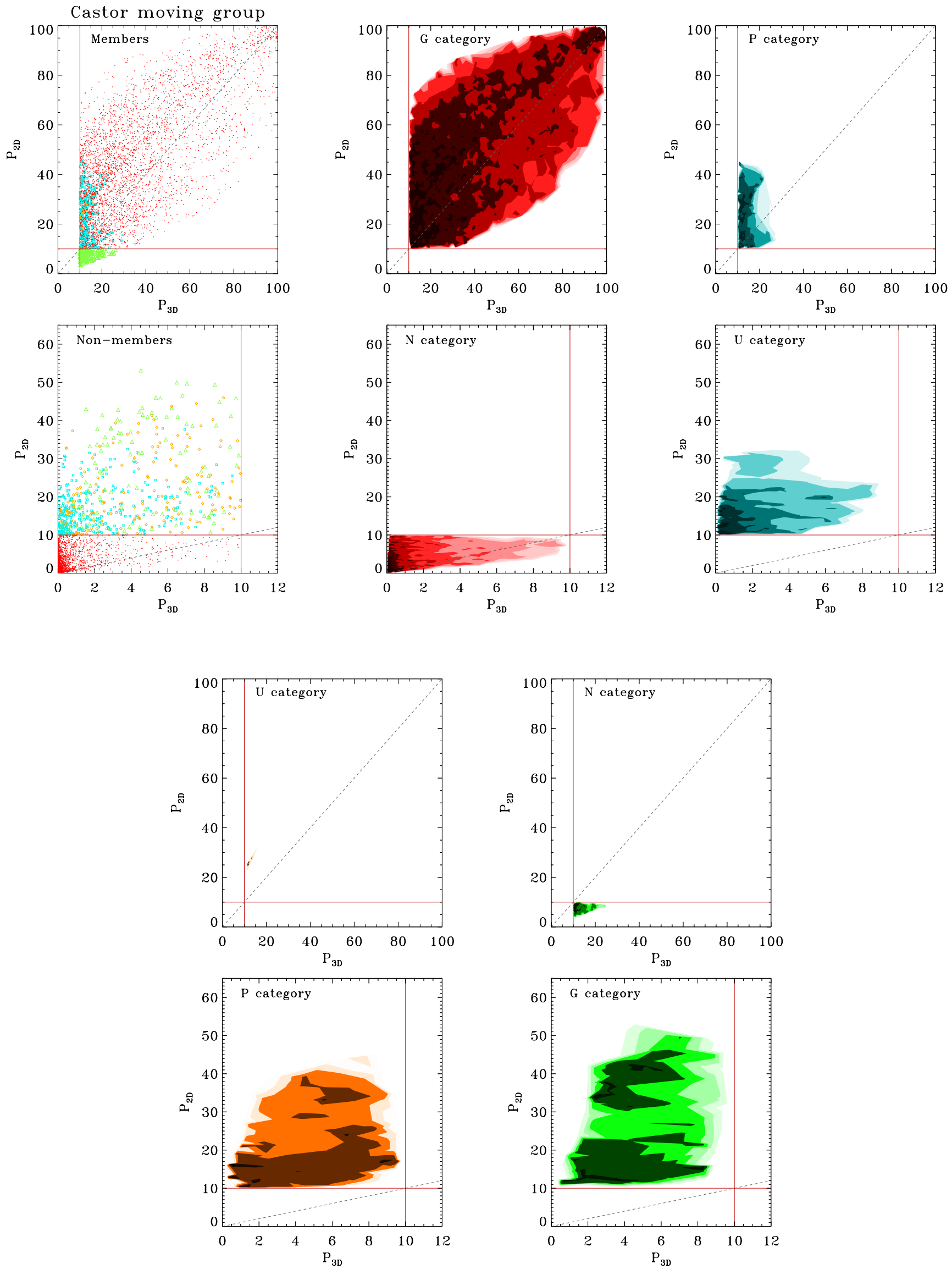

Fig. B.3. As Fig. B.1 but for the Castor moving group. 
A\&A 567, A52 (2014)
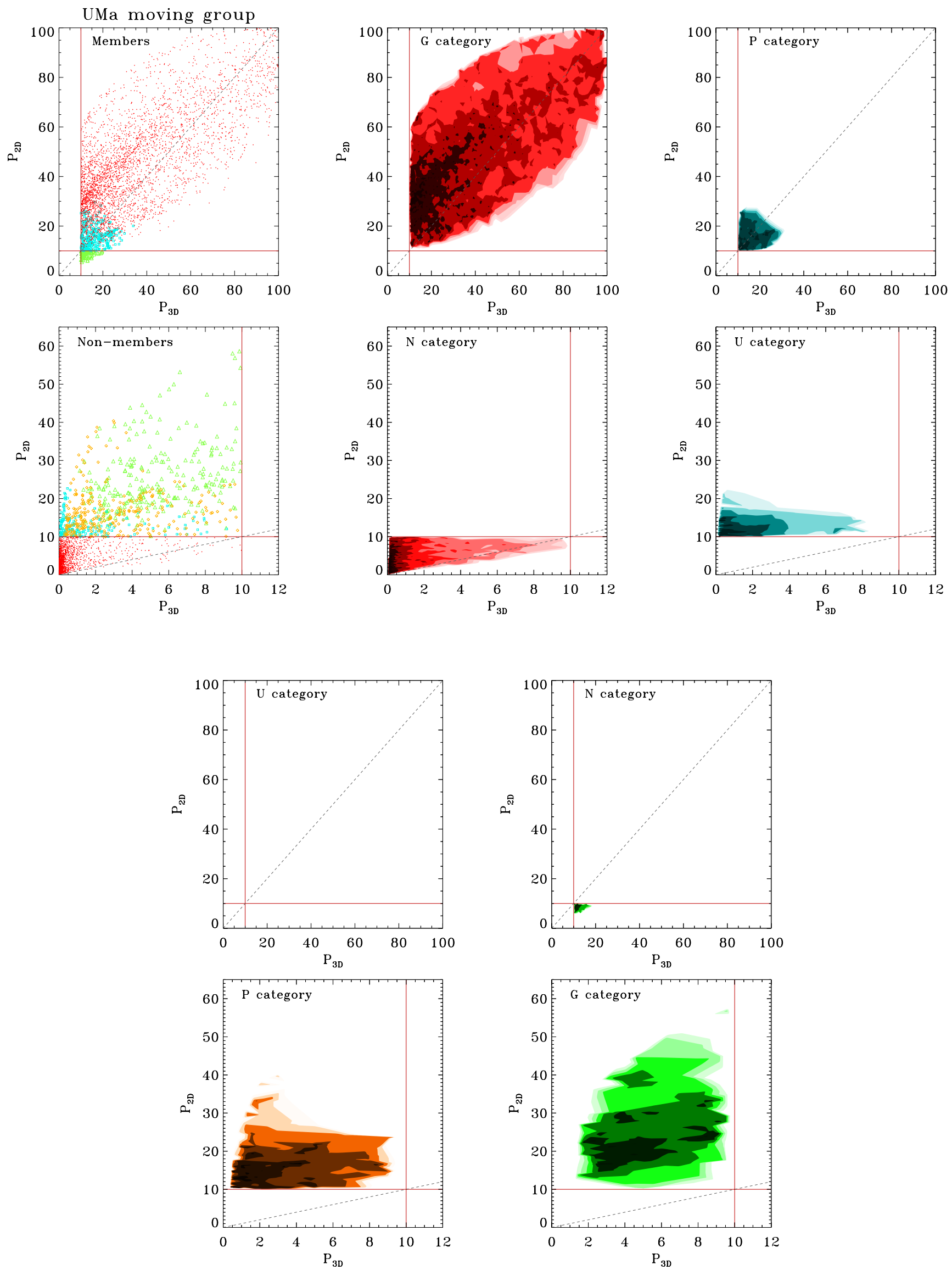

Fig. B.4. As Fig. B.1 but for the UMa moving group. 
A. Klutsch et al.: Reliable probabilistic determination of membership in stellar kinematic groups in the young disk


Fig. B.5. As Fig. B.1 but for the Hyades supercluster. 

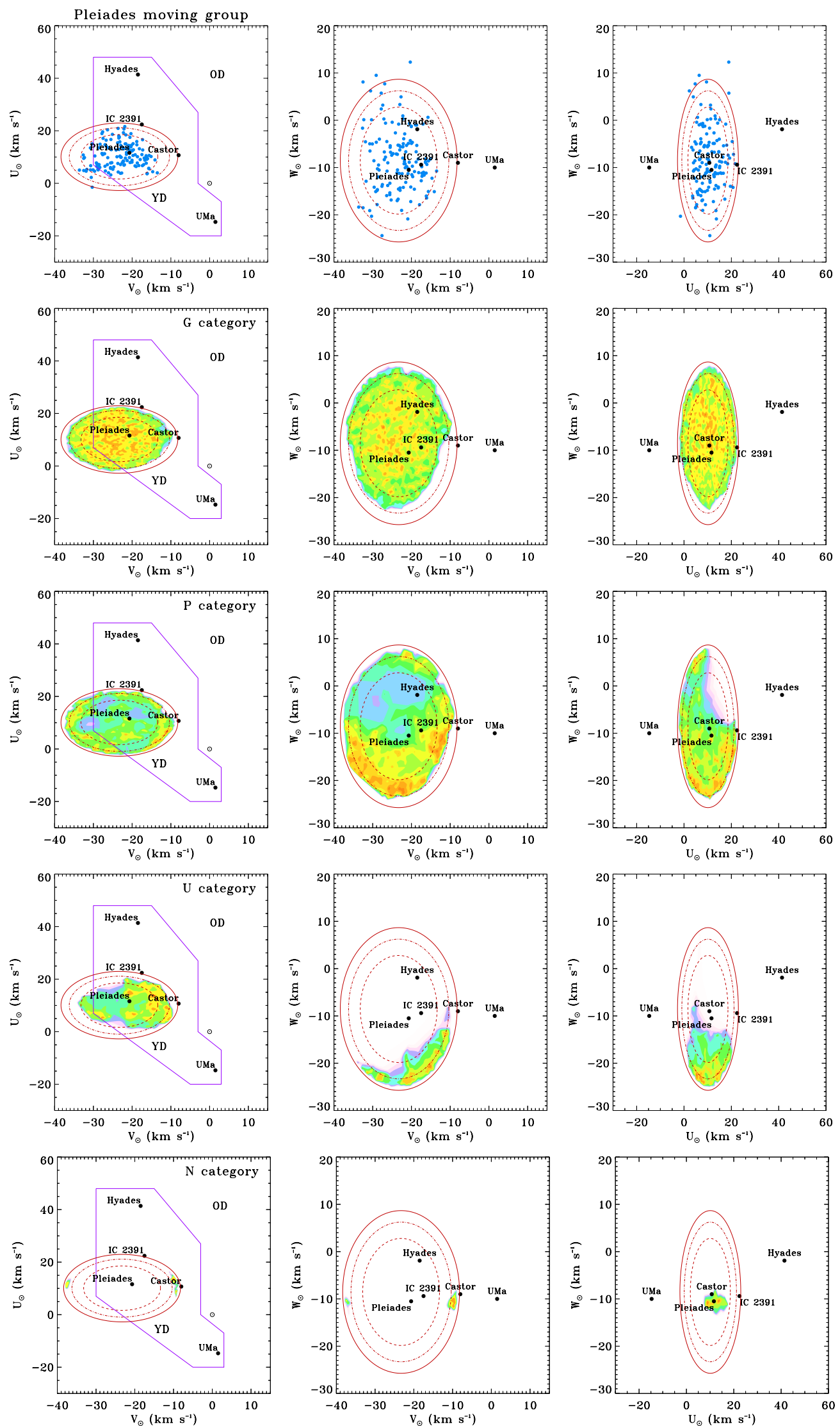

Fig. B.6. $(U, V),(V, W)$ and $(U, W)$ planes (from left to right) of the member candidates to the Pleiades MG, which are selected by means of Monte Carlo simulations. From the second row to the bottom, we display the distribution of good, possible, unlikely and disregarded candidates. The color codes the density level: light pink/purple when the sources are sparsely distributed, to orange/red for a higher density. For comparison purposes, we show the loci of known members taken from Montes et al. (2001) on the first row. We also over-plotted ellipses of three $k$ values, which correspond to the 10\% threshold for 1D, 2D, and 3D spatial considerations (Fig. A.2) by dashed, dash-dotted, and solid lines, respectively. 
A. Klutsch et al.: Reliable probabilistic determination of membership in stellar kinematic groups in the young disk
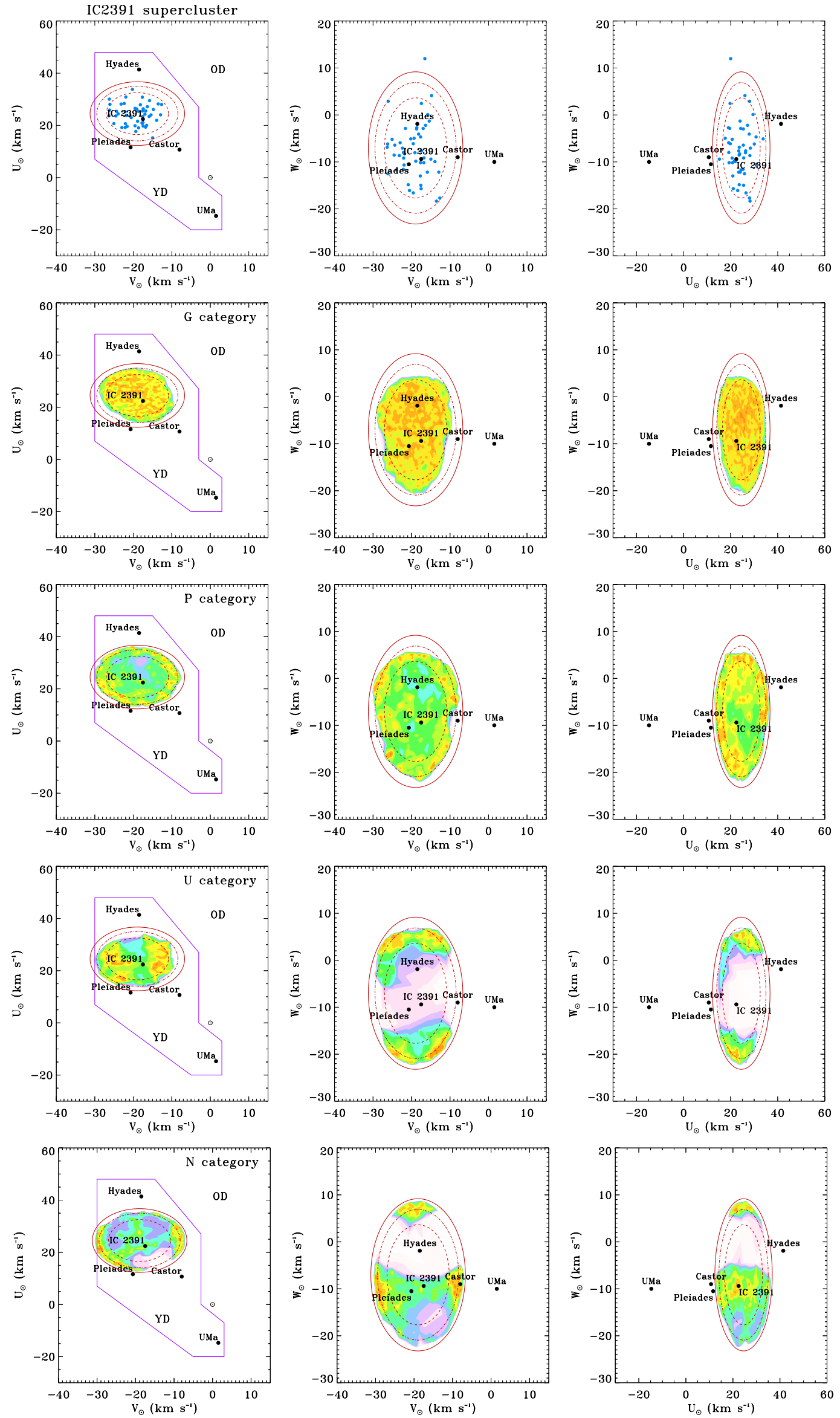

Fig. B.7. As Fig. B.6 but for the IC 2391 supercluster. 
A\&A 567, A52 (2014)
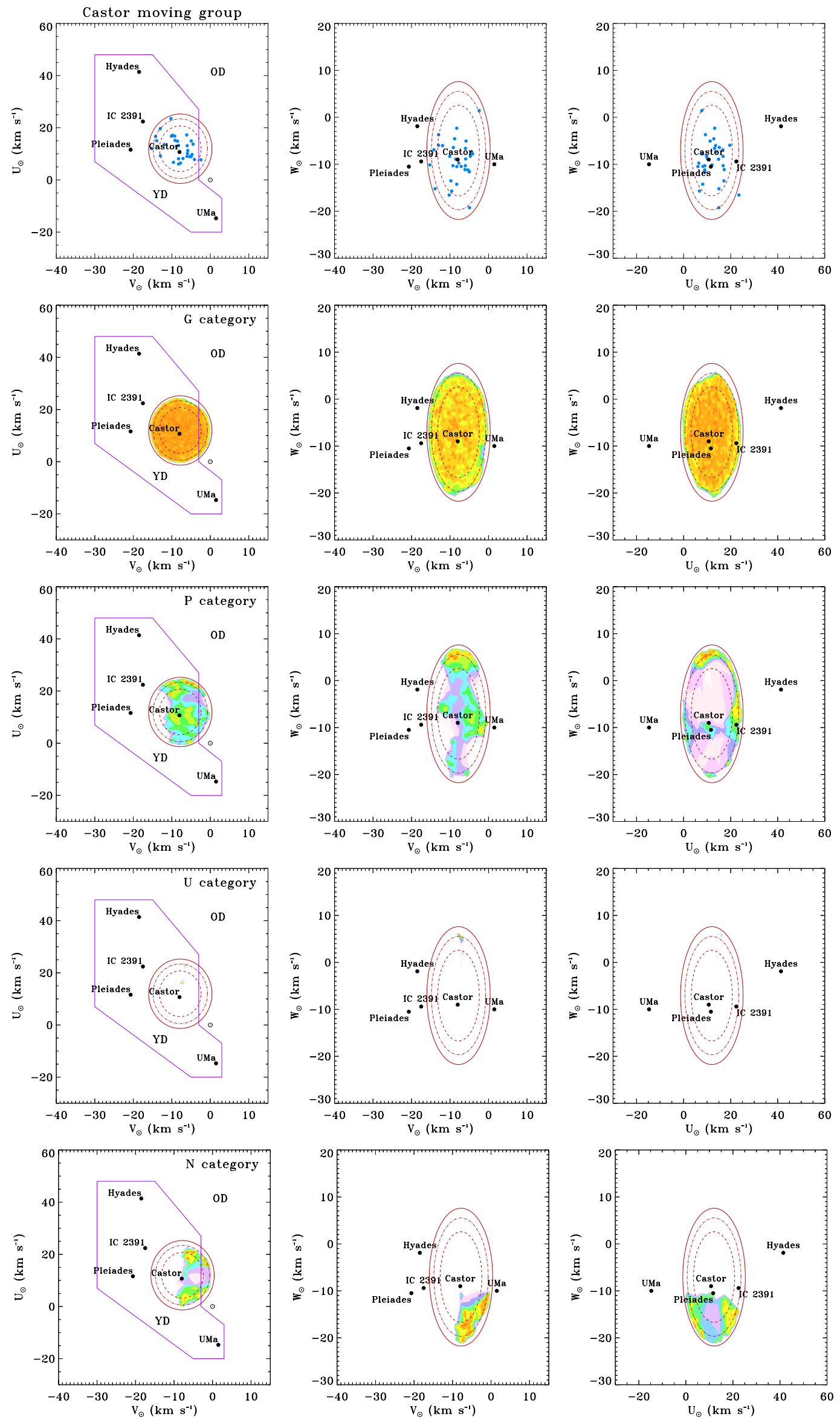

Fig. B.8. As Fig. B.6 but for the Castor moving group. 
A. Klutsch et al.: Reliable probabilistic determination of membership in stellar kinematic groups in the young disk
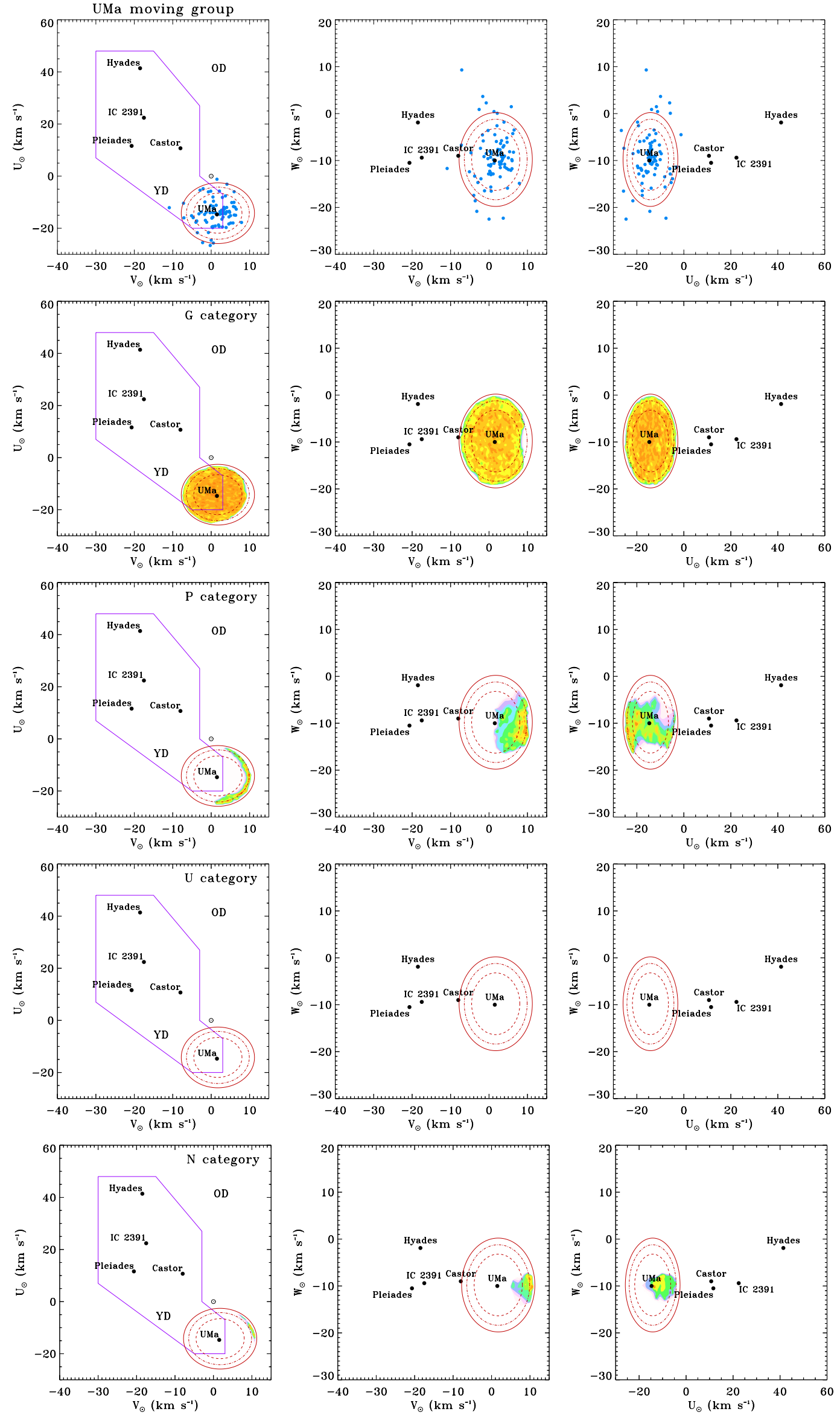

Fig. B.9. As Fig. B.6 but for the UMa moving group. 
A\&A 567, A52 (2014)
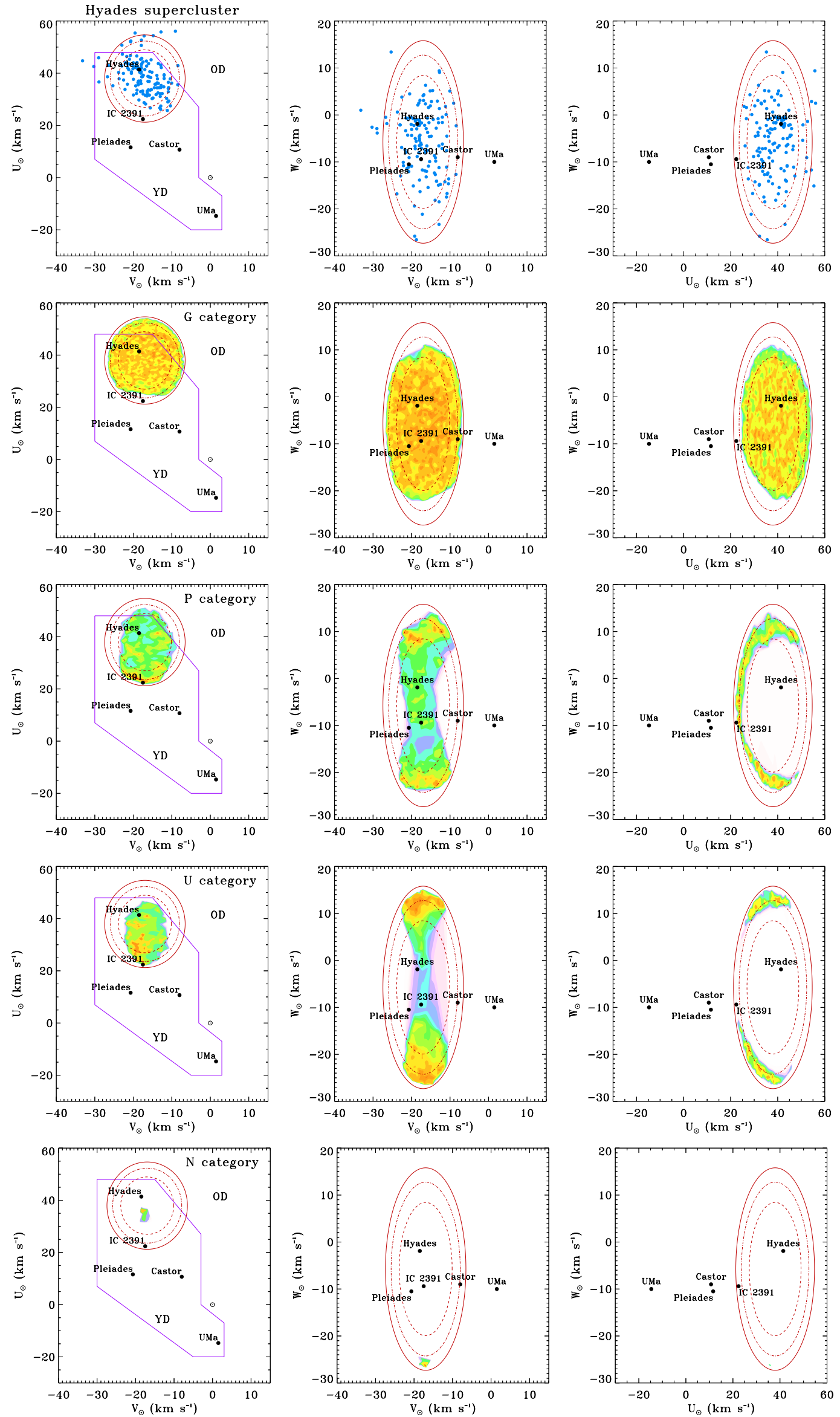

Fig. B.10. As Fig. B.6 but for the Hyades supercluster. 